This is the peer reviewed version of the following article: Ye, M., Anderson, B. D. O., and Yu, C. (2017) Distributed modelindependent consensus of Euler-Lagrange agents on directed networks. Int. J. Robust. Nonlinear Control, 27: 2428- 2450, which has been published in final form at 10.1002/rnc.3689. This article may be used for non-commercial purposes in accordance with Wiley Terms and Conditions for Use of Self-Archived Versions.

\title{
Distributed model-independent consensus of Euler-Lagrange agents on directed networks
}

\author{
Mengbin Ye, Brian D.O. Anderson, Changbin Yu* \\ Hangzhou Dianzi University, Hangzhou, Zhejiang, China \\ Research School of Engineering, Australian National University, Canberra A.C.T., Australia \\ Data61-CSIRO (formerly NICTA), Canberra, A.C.T., Australia
}

\begin{abstract}
SUMMARY
This paper proposes a distributed model-independent algorithm to achieve leaderless consensus on a directed network where each fully-actuated agent has self-dynamics described by Euler-Lagrange equations of motion. Specifically, we aim to achieve consensus of the generalised coordinates with zero generalised velocity. We show that on a strongly connected graph, a model-independent algorithm can achieve the consensus objective at an exponential rate if an upper bound on the initial conditions is known a priori. By model-independent, we mean that each agent can execute the algorithm with no knowledge of the equations describing the self-dynamics of any agent. For design of the control laws which achieve consensus, a control gain scalar and a control gain matrix are required to satisfy several inequalities involving bounds on the matrices of the agent dynamic model, bounds on the Laplacian matrix describing the network topology and the set of initial conditions; design of the algorithm therefore requires some knowledge on the bounds of the agent dynamical parameters. Because only bounds are required, the proposed algorithm offers robustness to uncertainty in the parameters of the multiagent system. We systematically show that additional relative velocity information improves the performance of the controller. Numerical simulations are provided to show the effectiveness of the algorithm.
\end{abstract}

KEY WORDS: model-independent, Euler-Lagrange system, semi-global, directed graph, leaderless consensus

\section{INTRODUCTION}

Coordination and control of autonomous, cooperating agents to achieve a common objective is an area of research which has grown in significance over the last two decades. A group of interacting agents is collectively called a multiagent system, and in certain applications, a correctly coordinated multiagent system offers advantages over a single complex agent. The term "agent" can be applied to many different controllable subsystems and as a result, multiagent systems research has a broad range of applications. Recent topics on cooperative multiagent systems are surveyed in $[1,2]$.

Consensus problems are an important and widely studied subset of multiagent coordination problems. The leaderless consensus problem studies how a network of agents can be controlled to reach agreement on a commonly defined state value(s) by communication and negotiation between neighbours. Focus is placed on developing distributed control laws which allow consensus to be achieved; by distributed we mean that each agent can execute the control law without requiring information about the network as a whole, see [3]. Apparently, the topological constraints of the network are linked to the self-dynamics of agents when it comes to studying control laws which guarantee consensus. Comprehensive treatment of consensus problems for agents with single-, double-integrator and linear self-dynamics, for a wide range of topological constraints can be found in $[3,4]$. The surveys $[1,2]$ cover consensus problems for agents with general nonlinear dynamics.

${ }^{*}$ Correspondence to: School of Automation, Hangzhou Dianzi University, Hangzhou 310018, China. Email: Brad.Yu@anu.edu.au 
The nonlinear Euler-Lagrange equations of motion can be used to model the dynamics of a large class of mechanical, electrical and electromechanical systems. As such, multiagent coordination problems where each agent has self-dynamics described by Euler-Lagrange equations are well motivated. Some existing results have studied control laws which utilise exact knowledge of the Euler-Lagrange equations describing the agent dynamics [5, 6, 7]. Euler-Lagrange equations can also be linearly parametrised $[8,9]$. This linear parametrisation can be used in adaptive algorithms to estimate uncertain agent parameters. Containment control, a variation of trajectory tracking, using adaptive algorithms is studied in [10,11,12]. Leaderless consensus is also studied in [11]. Adaptive tracking algorithms are studied in $[13,14,15,16,17]$. Rendezvous to a stationary leader using adaptive control with collision avoidance capabilities is proposed in [18].

In comparison, there have been relatively few works studying model-independent algorithms, i.e. algorithms for obtaining robust controllers. Almost all existing results assume neighbouring agent interactions are modelled by an undirected graph. The pioneering work in [19] considered leaderless position consensus on an undirected graph. When each agent has an individual target set of generalised coordinates, consensus to the intersection of the target sets is studied on an undirected graph in [20]. Consensus on an undirected network in the presence of time-delays is studied in [21]. In [22], flocking is achieved assuming an undirected graph. Tracking of a leader with nonconstant velocity where the subgraph of followers is undirected is studied in [23, 24, 25, 26]. Robust containment control for an undirected follower subgraph is considered in [27]. Rendezvous to a stationary leader with collision avoidance is studied on an undirected graph in [28]. Directed graphs representing unilateral information flow are seen as more desirable than undirected graphs (i.e. bilateral information flow) from the following two points of view. Firstly, a directed network allows for agents with heterogeneous sensing and/or communicating capabilities (e.g. different sensing radius). Secondly, a directed graph allows each agent to reduce its sensing/communicating burden by reducing the number of neighbouring agents. The passivity analysis in [29] showed synchronisation of the velocities (but not the positions) on strongly connected graphs. Rendezvous to a stationary leader is studied on a directed spanning tree in [30]. Tracking of a moving leader on a directed spanning tree is studied in [26], but restrictive constraints are imposed on the leader's trajectory.

Further study of model-independent algorithms is desirable for several reasons. In the context of adaptive algorithms, given a unique Euler-Lagrange equation, determining the minimum number of parameters required is difficult in general [8]. The linear parametrisation also requires knowledge of the exact equation structure. This means that the adaptive algorithms cannot cope with unmodelled agent dynamics. By their definition, the same form of a model-independent controller can be applied to various agents with minor alterations. Model-independent algorithms are reminiscent of robust controllers; stability is guaranteed given limited knowledge of upper bounds on parameters of the multiagent system $[21,30]$.

In [30], and as we show in this paper, model-independent controllers can achieve the control objective exponentially fast, with a computable minimum rate of convergence. Exponentially stable systems are desired over systems which are asymptotically stable, but not exponentially so, because they offer better rejection to noise and disturbances. Adaptive controllers will yield exponential stability if certain conditions, e.g. persistency of excitation, are satisfied. However, satisfaction of these conditions has not been verified for most existing works studying adaptive controllers in EulerLagrange networks.

\subsection{Contributions and Paper Structure}

The key contribution of this paper is to show that a network of heterogeneous Euler-Lagrange agents can achieve leaderless consensus if the interaction topology is strongly connected. While several model-independent results exist for leader-follower consensus on directed graphs, to date, no results are available for model-independent algorithms achieving leaderless position consensus. The algorithm studied in this paper requires knowledge of the graph topology. In addition, a control gain scalar and a control gain matrix, used by every agent must satisfy a set of lower bounding inequalities. These inequalities require some limited knowledge of the bounds on the agent dynamic parameters, limited knowledge of the network topology and an upper bound on the 
initial conditions (which may be arbitrarily large). The last requirement means the algorithm is semi-globally stable. In other words, a bound on initial conditions simply requires recomputation of the control gain and stability is assured. Design of the algorithm is therefore centralised, but the algorithm is distributed when executed. While this paper draws some intellectual insights from [30], the problem of rendezvous to a stationary leader, and not the problem of consensus, is studied in [30]. The findings in [30] concerning control gain design and semi-global stability are consistent with what will be presented in this paper. Furthermore, we show that additional relative velocity information can be utilised to systematically achieve faster convergence. The exponential stability of the algorithm proposed in this paper is a desirable advantage over the model-independent algorithms in $[19,21,25,26,27,28]$, which do not have the property of being exponentially stable.

The paper structure is as follows. Section 2 provides the mathematical background required to analyse the proposed algorithm. At the same time a formal definition of the leaderless consensus problem is provided. The algorithm and stability proof are detailed in Section 3. Section 4 provides simulations to show the effectiveness of the proposed algorithm, and the paper is concluded in Section 5.

\section{BACKGROUND AND PROBLEM STATEMENT}

\subsection{Mathematical Notation and Matrix Theory}

We begin by providing definitions of notation and several lemmas and theorems for later use. We use $\otimes$ to denote the Kronecker product, refer to [31] for the properties of Kronecker products. The $p \times p$ identity matrix is $\boldsymbol{I}_{p}$ and the $n \times 1$ column vector of all ones (respectively all zeros) as $\mathbf{1}_{n}$ (respectively $\mathbf{0}_{n}$ ). The Euclidean norm of a vector is denoted by $\|\cdot\|_{2}$. For a square matrix $\boldsymbol{A}$, the spectral norm is the matrix norm induced by the Euclidean vector norm and denoted as $\|\boldsymbol{A}\|_{2}$. The properties of the spectral norm will be frequently used in this paper, see [31] for details. A symmetric matrix $\boldsymbol{A} \in \mathbb{R}^{n \times n}$ which is positive definite (respectively nonnegative definite) is denoted by $\boldsymbol{A}>0$ (respectively $\boldsymbol{A} \geq 0$ ). We order the eigenvalues of $\boldsymbol{A}$ as $\lambda_{1}(\boldsymbol{A})=\lambda_{\min }(\boldsymbol{A}) \leq$ $\lambda_{2}(\boldsymbol{A}) \leq \ldots \leq \lambda_{n-1}(\boldsymbol{A}) \leq \lambda_{n}(\boldsymbol{A})=\lambda_{\max }(\boldsymbol{A})$. For two symmetric matrices $\boldsymbol{A}, \boldsymbol{B}$, the expression $\boldsymbol{A}>\boldsymbol{B}$ is equivalent to $\boldsymbol{A}-\boldsymbol{B}>0$. The following eigenvalue inequality expressions will be frequently used in the paper.

$$
\begin{aligned}
\lambda_{\min }(\boldsymbol{A}) & >\lambda_{\max }(\boldsymbol{B}) \Rightarrow \boldsymbol{A}>\boldsymbol{B} \\
\lambda_{\max }(\boldsymbol{A}+\boldsymbol{B}) & \leq \lambda_{\max }(\boldsymbol{A})+\lambda_{\max }(\boldsymbol{B}) \\
\lambda_{\min }(\boldsymbol{A}+\boldsymbol{B}) & \geq \lambda_{\min }(\boldsymbol{A})+\lambda_{\min }(\boldsymbol{B}) \\
\lambda_{\min }(\boldsymbol{A}) \boldsymbol{x}^{\top} \boldsymbol{x} & \leq \boldsymbol{x}^{\top} \boldsymbol{A} \boldsymbol{x} \leq \lambda_{\max }(\boldsymbol{A}) \boldsymbol{x}^{\top} \boldsymbol{x}
\end{aligned}
$$

Theorem 1 ([31])

Consider a symmetric block matrix, partitioned as

$$
A=\left[\begin{array}{cc}
B & C \\
C^{\top} & D
\end{array}\right]
$$

Then $\boldsymbol{A}>0$ if and only if $\boldsymbol{B}>0$ and $\boldsymbol{D}-\boldsymbol{C}^{\top} \boldsymbol{B}^{-1} \boldsymbol{C}>0$. Equivalently, $\boldsymbol{A}>0$ if and only if $\boldsymbol{D}>0$ and $\boldsymbol{B}-\boldsymbol{C} \boldsymbol{D}^{-1} \boldsymbol{C}^{\top}>0$.

Lemma 1

Let a quadratic function with arguments $\boldsymbol{x}, \boldsymbol{y} \in \mathbb{R}^{n}$ be expressed as

$$
W=\left[\begin{array}{l}
\boldsymbol{x} \\
\boldsymbol{y}
\end{array}\right]^{\top}\left[\begin{array}{cc}
\boldsymbol{B} & \boldsymbol{C} \\
\boldsymbol{C}^{\top} & \boldsymbol{D}
\end{array}\right]\left[\begin{array}{l}
\boldsymbol{x} \\
\boldsymbol{y}
\end{array}\right], \text { with }\left[\begin{array}{cc}
\boldsymbol{B} & \boldsymbol{C} \\
\boldsymbol{C}^{\top} & \boldsymbol{D}
\end{array}\right]>0
$$

Define $\boldsymbol{G}:=\boldsymbol{D}-\boldsymbol{C}^{\top} \boldsymbol{B}^{-1} \boldsymbol{C}$. Then there holds

$$
\lambda_{\min }(\boldsymbol{G}) \boldsymbol{y}^{\top} \boldsymbol{y} \leq \boldsymbol{y}^{\top} \boldsymbol{G} \boldsymbol{y} \leq W
$$


Proof

The proof is immediate obtained by recalling Theorem 1 and observing that

$$
W=\boldsymbol{y}^{\top} \boldsymbol{G} \boldsymbol{y}+\left[\boldsymbol{y}^{\top} \boldsymbol{C}^{\top} \boldsymbol{B}^{-1}+\boldsymbol{x}^{\top}\right] \boldsymbol{B}\left[\boldsymbol{B}^{-1} \boldsymbol{C} \boldsymbol{y}+\boldsymbol{x}\right]
$$

Theorem 2 ([31])

Let $\boldsymbol{A} \in \mathbb{R}^{n \times n}$ be symmetric and partitioned as

$$
\boldsymbol{A}=\left[\begin{array}{cc}
B & C \\
C^{\top} & D
\end{array}\right]
$$

with $\boldsymbol{B} \in \mathbb{R}^{m \times m}, \boldsymbol{D} \in \mathbb{R}^{(n-m) \times(n-m)}, \boldsymbol{C} \in \mathbb{R}^{m \times(n-m)}$. Then $\lambda_{i}(\boldsymbol{A}) \leq \lambda_{i}(\boldsymbol{D}) \leq \lambda_{i+m}(\boldsymbol{A})$.

Lemma 2

Let $g(x, y)$ be a function given as

$$
g(x, y)=a x^{2}+b y^{2}-c x y^{2}-d x y
$$

for real positive scalars $a, c, d>0$. Then for a given $\mathcal{Y}>0$, there exist $b>0$ such that $g(x, y)$ is positive definite for all $y \in[0, \mathcal{Y}]$ and $x \in[0, \infty)$.

Proof

Observe that $\frac{\partial g}{\partial x}=2 a x-c y^{2}-d y$. For some fixed value $y$, the minimum of $g(x, y)$ is at $x=$ $\frac{c y^{2}+d y}{2 a}:=z(y)$. Note that this point is a minimum because $\frac{\partial^{2} g}{\partial x^{2}}=2 a>0$. Define the new function $h(y):=g(z(y), y)$, and observe that

$$
\begin{aligned}
h(y) & =z(y)^{2} a+y^{2} b-z(y) c y^{2}-z(y) d y \\
& =\frac{1}{4 a} y^{2}\left(4 a b-c^{2} y^{2}-d^{2}-2 c d y\right)
\end{aligned}
$$

It follows that

$$
b>\frac{c^{2} \mathcal{Y}^{2}+d^{2}+2 c d \mathcal{Y}}{4 a}
$$

implies $4 a b-c^{2} y^{2}-d^{2}-2 c d y>0$ for $y \in[0, \mathcal{Y}]$, which in turn yields $h(y)>0$ for $y \in[0, \mathcal{Y}]$. The definition of $h(y)$ then yields the lemma statement.

\subsection{Graph Theory}

The information flow between agents can be modelled by a weighted directed graph, given as $\mathcal{G}=(\mathcal{V}, \mathcal{E}, \mathcal{A})$, with the finite, nonempty set of nodes $\mathcal{V}=\left\{v_{1}, \ldots, v_{n}\right\}$ with node indices $\mathcal{I}=$ $\{1,2, \ldots, n\}$, and with a corresponding set of ordered edges $\mathcal{E} \subseteq \mathcal{V} \times \mathcal{V}$. We denote an ordered edge of $\mathcal{G}$ as $e_{i j}=\left(v_{i}, v_{j}\right)$ and the assumption $e_{i j}=e_{j i}$ does not hold in general because $\mathcal{G}$ is a directed graph. The edge $e_{i j}=\left(v_{i}, v_{j}\right)$ is said to be outgoing with respect to $v_{i}$ and incoming with respect to $v_{j}$. In other words, an edge $\left(v_{i}, v_{j}\right)$ indicates that $v_{j}$ obtains information about $v_{i}$ (the precise nature of the information is detailed in Section 2.4). The weighted adjacency matrix $\mathcal{A} \in \mathbb{R}^{n \times n}$ of $\mathcal{G}$ has nonnegative elements $a_{i j}$. The elements of $\mathcal{A}$ are defined such that $a_{i j}>0 \Leftrightarrow e_{j i} \in \mathcal{E}$ while $a_{i j}=0$ if $e_{j i} \notin \mathcal{E}$ and it is assumed $a_{i i}=0, \forall i$. The neighbour set of $v_{i}$ is denoted by $\mathcal{N}_{i}=\left\{v_{j} \in \mathcal{V}:\left(v_{j}, v_{i}\right) \in \mathcal{E}\right\}$. The $n \times n$ Laplacian matrix, $\mathcal{L}=\left\{l_{i j}\right\}$, of the associated digraph $\mathcal{G}$ is defined as

$$
l_{i j}= \begin{cases}\sum_{k=1, k \neq i}^{n} a_{i k} & \text { for } j=i \\ -a_{i j} & \text { for } j \neq i\end{cases}
$$

A directed path is a sequence of edges of the form $\left(v_{p_{1}}, v_{p_{2}}\right),\left(v_{p_{2}}, v_{p_{3}}\right), \ldots$, where $v_{p_{i}} \in \mathcal{V}, e_{i j} \in \mathcal{E}$. Node $i$ is reachable from node $j$ if there exists a directed path from $v_{j}$ to $v_{i}$. A graph is said to be strongly connected if every node is reachable from every other node.

For use in the sequel we now provide a key lemma and the definitions of a certain projection matrix and orthonormal matrix. 
Lemma 3 ([32])

Let $\mathcal{G}$ be a strongly connected directed graph, with associated Laplacian $\mathcal{L}$. Denote $\gamma=\left[\gamma_{1}, \ldots, \gamma_{n}\right]^{\top}$ as the unique left eigenvector of $\mathcal{L}$ associated with the simple zero eigenvalue, with $\gamma_{i}>0, \forall i$ and note that there holds $\boldsymbol{\gamma}^{\top} \mathbf{1}_{n}=1$. Let $\boldsymbol{\Gamma}=\operatorname{diag}\left\{\gamma_{1}, \ldots, \gamma_{n}\right\}$. Then

$$
\boldsymbol{\Gamma} \mathcal{L}+\mathcal{L}^{\top} \boldsymbol{\Gamma}:=\widetilde{\mathcal{L}} \geq 0
$$

Because the elements of $\gamma$ are strictly positive and $\gamma^{\top} \mathbf{1}=1$, it follows that $\lambda_{i}(\boldsymbol{\Gamma})<1, \forall i$ and thus $\|\boldsymbol{\Gamma}\|_{2}<1$. Observe that $\mathbf{1}^{\top} \boldsymbol{\Gamma} \mathcal{L}=\boldsymbol{\gamma}^{\top} \mathcal{L}=\mathbf{0}$ and $\boldsymbol{\Gamma} \mathcal{L} \mathbf{1}=\mathbf{0}$. This implies that $\boldsymbol{\Gamma} \mathcal{L}$ is the Laplacian of a balanced and strongly connected graph, and thus $\widetilde{\mathcal{L}}$ is the Laplacian of an undirected connected graph [33]. For future use, we order the eigenvalues of $\widetilde{\mathcal{L}}$ as in Section 2.1: $\lambda_{1}(\widetilde{\mathcal{L}})=0<\lambda_{2}(\widetilde{\mathcal{L}}) \leq$ $\ldots \leq \lambda_{n}(\widetilde{\mathcal{L}})$.

2.2.1. Projection Matrix For future use, we define the projection matrix $\boldsymbol{Z} \in \mathbb{R}^{n \times n}$ as:

$$
\boldsymbol{Z}=\boldsymbol{I}_{n}-\frac{1}{n} \mathbf{1 1}^{\top}
$$

where $n$ corresponds to the number of agents in the network. Now observe the following useful properties. Since $\boldsymbol{Z}$ is a projection matrix, it is idempotent; $\boldsymbol{Z}=\boldsymbol{Z}^{\top}=\boldsymbol{Z}^{2}$. Notice that the row and column sums of $\boldsymbol{Z}$ are equal to zero; $\boldsymbol{Z}$ has a single eigenvalue at 0 with corresponding eigenvector $\mathbf{1}_{n}$. All other eigenvalues are equal to 1 . It can also be verified that $\mathcal{L} \boldsymbol{Z}=\mathcal{L}$ because each row sum of $\mathcal{L}$ is equal to zero. Furthermore, $\boldsymbol{Z} \widetilde{\mathcal{L}} \boldsymbol{Z}=\widetilde{\mathcal{L}}$ where $\widetilde{\mathcal{L}}$ is defined in Lemma 3. Further, let us define

$$
\boldsymbol{Z}_{m}=\boldsymbol{Z} \otimes \boldsymbol{I}_{m}
$$

Since both $\boldsymbol{Z}$ and $\boldsymbol{I}_{m}$ are idempotent, so is $\boldsymbol{Z}_{m}$ [34]. The properties of Kronecker products and the fact that any nonzero vector is an eigenvector of the identity matrix yield that the kernel of $\boldsymbol{Z}_{m}$ is spanned by $\mathbf{1} \otimes \boldsymbol{x}$ where $\boldsymbol{x} \in \mathbb{R}^{m}$. Furthermore, from the definition of the spectral norm it is observed that $\left\|\boldsymbol{Z}_{m}\right\|_{2}=1, \forall n, m$

2.2.2. Orthonormal Matrix For later use we define an orthonormal matrix $\boldsymbol{T} \in \mathbb{R}^{n \times n}$ with first column $\frac{1}{\sqrt{n}} \mathbf{1}_{n}$ as below:

$$
\boldsymbol{T}=\left[\frac{1}{\sqrt{n}} \mathbf{1}_{n}: \widetilde{\boldsymbol{T}}\right]
$$

Denote $\boldsymbol{T}_{p}=\boldsymbol{T} \otimes \boldsymbol{I}_{p}$ and $\widetilde{\boldsymbol{T}}_{p}=\widetilde{\boldsymbol{T}} \otimes \boldsymbol{I}_{p}$. By recognising the orthonormal properties of $\boldsymbol{T}$, observe that

$$
\boldsymbol{Z T}=\left[\boldsymbol{I}-\frac{1}{n} \mathbf{1 1}^{\top}\right]\left[\frac{1}{\sqrt{n}} \mathbf{1}_{n} \vdots \widetilde{\boldsymbol{T}}\right]=\left[\mathbf{0}_{n} \vdots \widetilde{\boldsymbol{T}}\right]
$$

For some $\boldsymbol{q} \in \mathbb{R}^{n p}$, let $\boldsymbol{q}=\boldsymbol{T}_{p} \overline{\boldsymbol{q}}$ (or $\overline{\boldsymbol{q}}=\boldsymbol{T}_{p}^{\top} \boldsymbol{q}$ ). Then

$$
\boldsymbol{Z}_{p} \boldsymbol{q}=\left(\left[\mathbf{0}_{n}: \widetilde{\boldsymbol{T}}\right] \otimes \boldsymbol{I}_{p}\right) \overline{\boldsymbol{q}}
$$

Let $\widetilde{\boldsymbol{q}}=\left[\bar{q}_{(p+1)}, \bar{q}_{(p+2)}, \ldots, \bar{q}_{n p}\right]^{\top}$ i.e. $\widetilde{\boldsymbol{q}}=\overline{\boldsymbol{q}}$ with the first $p$ entries removed. Observe that

$$
\left(\widetilde{\boldsymbol{T}} \otimes \boldsymbol{I}_{p}\right) \widetilde{\boldsymbol{q}}=\left(\left[\mathbf{0}_{n} \vdots \widetilde{\boldsymbol{T}}\right] \otimes \boldsymbol{I}_{p}\right) \overline{\boldsymbol{q}}=\boldsymbol{Z}_{p} \boldsymbol{q}
$$

Three useful properties of $\widetilde{\boldsymbol{T}}_{p}$ are now given.

P1 For any vector $\boldsymbol{x} \in \mathbb{R}^{n p}$, and because $\widetilde{\boldsymbol{T}}^{\top} \widetilde{\boldsymbol{T}}=\boldsymbol{I}_{n-1}$, there holds $\left\|\widetilde{\boldsymbol{T}}_{p} \boldsymbol{x}\right\|_{2}=\sqrt{\boldsymbol{x}^{\top} \widetilde{\boldsymbol{T}}_{p}^{\top} \widetilde{\boldsymbol{T}}_{p} \boldsymbol{x}}=$ $\|\boldsymbol{x}\|_{2}$. 
P2 The identity $\boldsymbol{Z}=\widetilde{\boldsymbol{T}} \widetilde{\boldsymbol{T}}^{\top}$ follows by multiplying (11) on the right by $\boldsymbol{T}^{\top}$ to give

$$
\begin{aligned}
\boldsymbol{Z T \boldsymbol { T } ^ { \top }} & =\left[\mathbf{0}_{n} \vdots \widetilde{\boldsymbol{T}}\right]\left[\begin{array}{c}
\frac{1}{\sqrt{n}} \mathbf{1}_{n} \\
\cdots \\
\widetilde{\boldsymbol{T}}^{\top}
\end{array}\right] \\
\boldsymbol{Z} & =\widetilde{\boldsymbol{T}} \widetilde{\boldsymbol{T}}^{\top}
\end{aligned}
$$

Using Kronecker products, one also has $\boldsymbol{Z}_{p}=\widetilde{\boldsymbol{T}}_{p} \widetilde{\boldsymbol{T}}_{p}^{\top}$

P3 From property P1, and because projection matrices are contractive, we obtain the inequality $\left\|\widetilde{\boldsymbol{T}}_{p} \widetilde{\boldsymbol{q}}\right\|_{2}=\left\|\boldsymbol{Z}_{p} \boldsymbol{q}\right\|_{2}=\|\widetilde{\boldsymbol{q}}\|_{2} \leq\|\boldsymbol{q}\|_{2}$.

Lastly, we provide a lemma and a corollary for later use

Lemma 4

Let $\boldsymbol{A} \in \mathbb{R}^{n p \times n p}$ and $\boldsymbol{B} \in \mathbb{R}^{(n-1) p \times(n-1) p}$ be symmetric matrices, and let $\widetilde{\boldsymbol{T}}$ be defined as above. Then there holds

$$
\begin{gathered}
\lambda_{\min }(\boldsymbol{A}) \leq \lambda_{\min }\left(\widetilde{\boldsymbol{T}}_{p}^{\top} \boldsymbol{A} \widetilde{\boldsymbol{T}}_{p}\right) \\
\lambda_{\max }\left(\widetilde{\boldsymbol{T}}_{p}^{\top} \boldsymbol{A} \widetilde{\boldsymbol{T}}_{p}\right) \leq \lambda_{\max }(\boldsymbol{A}) \\
\lambda_{i}(\boldsymbol{B})=\lambda_{i}\left(\widetilde{\boldsymbol{T}}_{p} \boldsymbol{B} \widetilde{\boldsymbol{T}}_{p}^{\top}\right)
\end{gathered}
$$

Proof

Observe that

$$
\begin{aligned}
\boldsymbol{T}_{p}^{\top} \boldsymbol{A} \boldsymbol{T}_{p} & =\left(\left[\frac{1}{\sqrt{n}} \mathbf{1}: \widetilde{\boldsymbol{T}}\right] \otimes \boldsymbol{I}_{p}\right)^{\top} \boldsymbol{A}\left(\left[\frac{1}{\sqrt{n}} \mathbf{1}: \widetilde{\boldsymbol{T}}\right] \otimes \boldsymbol{I}_{p}\right) \\
& =\left[\begin{array}{cc}
\frac{1}{n}\left(\mathbf{1}^{\top} \otimes \boldsymbol{I}_{p}\right) \boldsymbol{A}\left(\mathbf{1} \otimes \boldsymbol{I}_{p}\right) & \frac{1}{\sqrt{n}}\left(\mathbf{1}^{\top} \otimes \boldsymbol{I}_{p}\right) \boldsymbol{A} \widetilde{\boldsymbol{T}}_{p} \\
\frac{1}{\sqrt{n}} \widetilde{\boldsymbol{T}}_{p}^{\top} \boldsymbol{A}\left(\mathbf{1} \otimes \boldsymbol{I}_{p}\right) & \widetilde{\boldsymbol{T}}_{p}^{\top} \boldsymbol{A} \widetilde{\boldsymbol{T}}_{p}
\end{array}\right]
\end{aligned}
$$

The expressions in (14) are then obtained using Theorem 2 and by observing that $\boldsymbol{T}_{p}^{\top} \boldsymbol{A} \boldsymbol{T}_{p}$ is similar to $\boldsymbol{A}$, i.e. they have the same eigenvalues (since $\boldsymbol{T}_{p}^{-1}=\boldsymbol{T}_{p}^{\top}$ ). Likewise, (15) can be obtained from the following similarity transformation

$$
\boldsymbol{T}_{p}\left[\begin{array}{cc}
0 & \mathbf{0}_{p \times(n-1) p} \\
\mathbf{0}_{(n-1) p \times p} & \boldsymbol{B}
\end{array}\right] \boldsymbol{T}_{p}^{\top}=\left[\begin{array}{cc}
0 & \mathbf{0}_{p \times(n-1) p} \\
\mathbf{0}_{(n-1) p \times p} & \widetilde{\boldsymbol{T}}_{p} \boldsymbol{B} \widetilde{\boldsymbol{T}}_{p}^{\top}
\end{array}\right]
$$

\section{Corollary 1}

Let $\widetilde{\boldsymbol{T}}$ be defined as above. Let $\widetilde{\mathcal{L}}$ be the Laplacian of an undirected, connected graph. Then the $n-1$ eigenvalues of $\widetilde{\boldsymbol{T}}^{\top} \widetilde{\mathcal{L}} \widetilde{\boldsymbol{T}}$ are the same as the $n-1$ nonzero eigenvalues of $\widetilde{\mathcal{L}}$. It follows that $\widetilde{\boldsymbol{T}}^{\top} \widetilde{\mathcal{L}} \widetilde{\boldsymbol{T}}>0$.

Proof

Recall firstly that for an undirected, connected graph, the associated symmetric Laplacian $\widetilde{\mathcal{L}}$ has a single eigenvalue at zero and all other eigenvalues are strictly positive. Furthermore, $\mathbf{1}_{n}^{\top} \widetilde{\mathcal{L}}=\widetilde{\mathcal{L}} \mathbf{1}_{n}=$ 
$\mathbf{0}_{n}$. From the fact that $\boldsymbol{T}^{\top}=\boldsymbol{T}^{-1}$, we conclude that $\widetilde{\mathcal{L}}$ is similar to $\boldsymbol{T}^{\top} \widetilde{\mathcal{L}} \boldsymbol{T}$. Observe that

$$
\begin{aligned}
& \boldsymbol{T}^{\top} \widetilde{\mathcal{L}} \boldsymbol{T}=\left[\frac{1}{\sqrt{n}} \mathbf{1}: \widetilde{\boldsymbol{T}}\right]^{\top} \widetilde{\mathcal{L}}\left[\frac{1}{\sqrt{n}} \mathbf{1}: \widetilde{\boldsymbol{T}}\right] \\
& =\left[\begin{array}{cc}
\frac{1}{n} \mathbf{1}_{n}^{\top} \widetilde{\mathcal{L}} \mathbf{1}_{n} & \frac{1}{\sqrt{n}} \mathbf{1}_{n}^{\top} \widetilde{\mathcal{L}} \widetilde{\boldsymbol{T}} \\
\frac{1}{\sqrt{n}} \widetilde{\boldsymbol{T}}^{\top} \widetilde{\mathcal{L}} \mathbf{1}_{n} & \widetilde{\boldsymbol{T}}^{\top} \widetilde{\mathcal{L}} \widetilde{\boldsymbol{T}}
\end{array}\right] \\
& =\left[\begin{array}{cc}
0 & \mathbf{0}_{n-1}^{\top} \\
\mathbf{0}_{n-1} & \widetilde{\boldsymbol{T}}^{\top} \widetilde{\mathcal{L}} \widetilde{\boldsymbol{T}}
\end{array}\right]
\end{aligned}
$$

Because of similarity, the eigenvalues of $\widetilde{\mathcal{L}}$ are equal to the eigenvalues of $\boldsymbol{T}^{\top} \widetilde{\mathcal{L}} \boldsymbol{T}$ (including their algebraic multiplicity). The corollary statement is then trivially obtained from expression (18).

\subsection{Euler-Lagrange Systems}

The dynamics of each agent in $\mathcal{G}$ is described using the nonlinear Euler-Lagrange equations, and the general form for the $i^{\text {th }}$ agent equation of motion is:

$$
\boldsymbol{M}_{i}\left(\boldsymbol{q}_{i}\right) \ddot{\boldsymbol{q}}_{i}+\boldsymbol{C}_{i}\left(\boldsymbol{q}_{i}, \dot{\boldsymbol{q}}_{i}\right) \dot{\boldsymbol{q}}_{i}=\boldsymbol{\tau}_{i}
$$

where $\boldsymbol{q}_{i} \in \mathbb{R}^{p}$ is a vector of the generalised coordinates, $\boldsymbol{M}_{i}\left(\boldsymbol{q}_{i}\right) \in \mathbb{R}^{p \times p}$ is the inertia matrix, $\boldsymbol{C}_{i}\left(\boldsymbol{q}_{i}, \dot{\boldsymbol{q}}_{i}\right) \in \mathbb{R}^{p \times p}$ is the Coriolis and centrifugal force matrix and $\boldsymbol{\tau}_{i} \in \mathbb{R}^{p}$ is the control input vector. Note that (19) is a subclass of Euler-Lagrange equations as it does not contain the vector of potential forces, commonly denoted as $\boldsymbol{g}_{i}\left(\boldsymbol{q}_{i}\right)$. This work assumes that all agents are fully-actuated. For each agent, let superscript $(j)$ denote the $j^{\text {th }}$ generalised coordinate; $\boldsymbol{q}_{i}=\left[q_{i}^{(1)}, \ldots, q_{i}^{(p)}\right]^{\top}$. ${ }^{\dagger}$ We assume that the systems described using (19) have the following properties given below (typical for this class of problems). See $[8,9]$ for details:

A1 The matrix $\boldsymbol{M}_{i}\left(\boldsymbol{q}_{i}\right)$ is symmetric positive definite.

A2 There exist scalar constants $k_{\underline{m}}, k_{\bar{M}}>0$ such that $k_{\underline{m}} \boldsymbol{I}_{p} \leq \boldsymbol{M}_{i}\left(\boldsymbol{q}_{i}\right) \leq k_{\bar{M}} \boldsymbol{I}_{p}, \forall i, \boldsymbol{q}_{i}$. It follows that $\sup _{\boldsymbol{q}_{i}}\left\|\boldsymbol{M}_{i}\right\|_{2} \leq k_{\bar{M}}$ and $k_{\underline{m}} \leq \inf _{\boldsymbol{q}_{i}}\left\|\boldsymbol{M}_{i}^{-1}\right\|_{2}{ }^{-1}$ for all $i$.

A3 The matrix $\boldsymbol{C}_{i}\left(\boldsymbol{q}_{i}, \dot{\boldsymbol{q}}_{i}\right)$ is defined such that $\dot{\boldsymbol{M}}_{i}-2 \boldsymbol{C}_{i}$ is skew-symmetric. It follows that $\dot{M}_{i}=C_{i}+C_{i}^{\top}$.

A4 There exists a scalar constant $k_{C}>0$ such that $\left\|\boldsymbol{C}_{i}\right\|_{2} \leq k_{C}\left\|\dot{\boldsymbol{q}}_{i}\right\|_{2}, \forall i, \dot{\boldsymbol{q}}_{i}$.

\subsection{Problem Statement}

The objective of this paper is to develop a model-independent, distributed algorithm which allows a directed network of Euler-Lagrange agents to achieve leaderless consensus of their generalised coordinates with zero generalised velocity. The leaderless consensus objective is achieved if:

$$
\begin{array}{cl}
\lim _{t \rightarrow \infty}\left\|\boldsymbol{q}_{i}(t)-\boldsymbol{q}_{j}(t)\right\|_{2}=0 & , \forall i, j=1, \ldots, n \\
\lim _{t \rightarrow \infty}\left\|\dot{\boldsymbol{q}}_{i}(t)\right\|_{2}=0 & , \forall i=1, \ldots, n
\end{array}
$$

The interaction between the agents is captured by the fixed, weighted, directed graph $\mathcal{G}$ with an associated Laplacian $\mathcal{L}$. Specifically, if for agent $v_{i}$ we have $a_{i j}>0$, then agent $i$ knows $a_{i j}$ and can obtain the quantity $\boldsymbol{q}_{i}-\boldsymbol{q}_{j}$, i.e. the relative generalised coordinates. The method by which $\boldsymbol{q}_{i}-\boldsymbol{q}_{j}$ is obtained is left open to interpretation and adaptation to the specific multiagent scenario. One can imagine robotic manipulators transmitting measured coordinates or vehicles measuring relative positions. In addition, we assume that agent $i$ can measure its own velocity $\dot{\boldsymbol{q}}_{i}$.

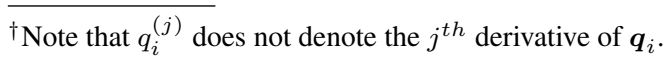


Finally, we make an assumption on the initial conditions. Specifically, let $\left\|\boldsymbol{q}(0)_{i}\right\|_{2} \leq k_{a} / \sqrt{n}$ and $\left\|\dot{\boldsymbol{q}}_{i}(0)\right\|_{2} \leq k_{b} / \sqrt{n}$ for all $i$, and where $n$ is the number of agents and $k_{a}, k_{b} \geq 0$ are known a priori. This is not unreasonable, as many systems will have an expected operating range for $\boldsymbol{q}$ and $\dot{\boldsymbol{q}}$ (in particular the initial conditions of (19)). This assumption on the initial conditions results in a semiglobally stable control algorithm; in the sequel, $k_{a}, k_{b}$ are used to compute the lower bounds on a control gain which ensures that the system trajectories are bounded by known values. We also show that for any given $k_{a}, k_{b}$ it is always possible to find a sufficiently large gain which ensures that the leaderless consensus objective is achieved.

By model-independent, we mean that the algorithm does not contain $\boldsymbol{M}_{i}, \boldsymbol{C}_{i} \forall i$ and does not contain the linear parametrisation. An agent's algorithm is distributed if, during execution, the agent only needs to receive information about its neighbours.

Notice that it is possible for $\boldsymbol{M}_{i} \neq \boldsymbol{M}_{j}$ and $\boldsymbol{C}_{i} \neq \boldsymbol{C}_{j}$ for any $i, j$ but $\boldsymbol{q}_{i} \in \mathbb{R}^{p}, \forall i$. In other words this work treats Euler-Lagrange agents which have heterogeneous parameters but with generalised coordinates which are defined such that $\boldsymbol{q}_{i}-\boldsymbol{q}_{j}, \forall i, j$ is meaningful.

\section{MAIN RESULT}

Consider the following model-independent algorithm for the $i^{t h}$ agent

$$
\tau_{i}=-\sum_{j \in \mathcal{N}_{i}} a_{i j}\left(\boldsymbol{q}_{i}-\boldsymbol{q}_{j}\right)-\mu \gamma_{i}^{-1} \boldsymbol{K}_{p} \dot{\boldsymbol{q}}_{i}
$$

where $a_{i j}$ is the weighted $(i, j)$ entry of the adjacency matrix $\mathcal{A}$ associated with the weighted directed graph $\mathcal{G}$. The control gain matrix $\boldsymbol{K}_{p} \in \mathbb{R}^{p \times p}$ is symmetric positive definite, and the control gain scalar $\mu$ is strictly positive. Both $\boldsymbol{K}_{p}$ and $\mu$ are universal for all agents. The terms $\gamma_{i}$ are the elements of $\gamma$ as defined in Lemma 3, and denote $\min _{i} \gamma_{i}=\gamma$ and $\max _{i} \gamma_{i}=\bar{\gamma}$. The terms $\mu$ and $\boldsymbol{K}_{p}$ are to be designed to satisfy several inequalities to ensure the control objective is achieved. Of course, $\mu$ and $\boldsymbol{K}_{p}$ can be combined to form a single positive definite matrix. However, we elect to keep them separate because, as will be seen below, it makes proof of stability intuitively easier. It is also straightforward to see that the algorithm (20) satisfies that model-independent requirements in Section 2.4. At the end of Section 3.2 we provide a step-by-step guide to designing $\mu, \boldsymbol{K}_{p}$.

For reasons which will become apparent in the proof, the matrix $\boldsymbol{K}_{p}$ is required to satisfy the following inequality

$$
\lambda_{\min }\left(\boldsymbol{K}_{p}\right)>\frac{\|\mathcal{L}\|_{2}}{2 \lambda_{2}(\widetilde{\mathcal{L}})}
$$

where $\widetilde{\mathcal{L}}$ is defined in Lemma 3, with $\mathcal{L}$ being the Laplacian associated with the strongly connected graph $\mathcal{G}$. From henceforth, we assume $\boldsymbol{K}_{p}$ has been designed to satisfy (21) and the primarily focus for the rest of the paper will be on deriving inequalities for $\mu$ which guarantee stability.

Let $\boldsymbol{q}=\left[\boldsymbol{q}_{1}^{\top}, \ldots, \boldsymbol{q}_{n}^{\top}\right]^{\top}$ be the stacked column vector of all $\boldsymbol{q}_{i}$. Consensus of the generalised coordinates can then be expressed as $\boldsymbol{q}=\mathbf{1}_{n} \otimes \boldsymbol{x}$ where $\boldsymbol{x} \in \mathbb{R}^{p}$ is the vector of final generalised coordinate values. Similarly, let $\dot{\boldsymbol{q}}=\left[\dot{\boldsymbol{q}}_{1}^{\top}, \ldots, \dot{\boldsymbol{q}}_{n}^{\top}\right]^{\top}$. Let $\boldsymbol{M}(\boldsymbol{q})=\operatorname{diag}\left[\boldsymbol{M}_{1}\left(\boldsymbol{q}_{1}\right), \ldots, \boldsymbol{M}_{n}\left(\boldsymbol{q}_{n}\right)\right]$, $\boldsymbol{C}(\boldsymbol{q}, \dot{\boldsymbol{q}})=\operatorname{diag}\left[\boldsymbol{C}_{1}\left(\boldsymbol{q}_{1}, \dot{\boldsymbol{q}}_{1}\right), \ldots, \boldsymbol{C}_{n}\left(\boldsymbol{q}_{n}, \dot{\boldsymbol{q}}_{n}\right)\right]$ and $\boldsymbol{K}=\boldsymbol{K}_{p} \otimes \boldsymbol{I}_{n}$. Since $\boldsymbol{M}_{i} \forall i$ and $\boldsymbol{K}_{p}$ are symmetric positive definite, then $\boldsymbol{M}$ and $\boldsymbol{K}$ are also symmetric positive definite. is

With this notation and applying control law (20) to each agent, the closed-loop networked system

$$
\boldsymbol{M}(\boldsymbol{q}) \ddot{\boldsymbol{q}}+\boldsymbol{C}(\boldsymbol{q}, \dot{\boldsymbol{q}}) \dot{\boldsymbol{q}}+\left(\mathcal{L} \otimes \boldsymbol{I}_{p}\right) \boldsymbol{q}+\mu \boldsymbol{\Gamma}^{-1} \boldsymbol{K} \dot{\boldsymbol{q}}=\mathbf{0}
$$

where $\Gamma$ was defined in Lemma 3, and expressed as the autonomous system

$$
\begin{aligned}
\dot{\boldsymbol{q}} & =\boldsymbol{v} \\
\dot{\boldsymbol{v}} & =-\boldsymbol{M}(\boldsymbol{q})^{-1}\left[\boldsymbol{C}(\boldsymbol{q}, \boldsymbol{v}) \boldsymbol{v}+\left(\mathcal{L} \otimes \boldsymbol{I}_{p}\right) \boldsymbol{q}+\mu \boldsymbol{\Gamma}^{-1} \boldsymbol{K} \boldsymbol{v}\right]
\end{aligned}
$$

One may trivially verify that the set of equilibrium points of (23) is given by $\mathcal{M}=\left\{\boldsymbol{q}, \boldsymbol{v}: \boldsymbol{q}=\mathbf{1}_{n} \otimes \boldsymbol{x}, \boldsymbol{v}=\mathbf{0}\right\}, \boldsymbol{x} \in \mathbb{R}^{p}$. 
Remark 1 (Previous model-independent controllers)

Controllers of the form (20) have been used to study consensus in undirected networks of EulerLagrange agents e.g. $[19,21]$. However, to the authors' knowledge, there have been no results studying leaderless consensus using the control law (20) on directed graphs. Because the Laplacian $\mathcal{L}$ is nonsymmetric for a directed graph, the analysis techniques in $[19,21]$ do not yield a stability result, and no apparent minor variation of the techniques would appear to suffice. One of the key contributions of this paper is to develop the analysis showing that the same controller can be extended to directed graphs. A second key contribution is to show that, for a sufficiently large control gain, convergence is in fact exponential. This exponential property is not proven in the undirected results of $[19,21]$.

Remark 2 (Synthesis of event-based controllers)

In the last few years, heavy focus has been placed on event-based controllers for coordinating multiagent systems [35] because of the practical advantages of event-based controllers. Event-based control of Euler-Lagrange networks has been relatively unstudied. In [36], an event-based adaptive controller is proposed but has the disadvantage of requiring continuous relative measurements $\boldsymbol{q}_{i}-\boldsymbol{q}_{j}$. In [37], a model-independent event-based controller is proposed for an undirected graph. As observed by the authors in recent preliminary investigations, simple model-independent controllers of the form in (20), and in [19, 30], have an advantage in the sense that they are easier to study in the event-based framework. This is in contrast to the complex model-independent controllers in $[25,26,27]$.

\subsection{An Upper Bound Using Initial Conditions}

Before we proceed with the main proof, a method is given to calculate a not necessarily tight upper bound on the initial states expressed as $\|\widetilde{\boldsymbol{q}}(0)\|_{2}<\mathcal{X}$ and $\|\dot{\boldsymbol{q}}(0)\|_{2}<\mathcal{Y}$ using the bounds on the initial condition, $k_{a}, k_{b}$. In the sequel, we show that these bounds hold for all time, and that as a consequence the leaderless consensus objective is achieved at an exponential rate. Due to spatial limitations, we only compute the bound on $\dot{\boldsymbol{q}}$ and leave the reader to follow an identical process for $\widetilde{\boldsymbol{q}}$. In keeping with the model-independent nature of the paper, define the following function

$$
\bar{V}_{\mu}=\left[\begin{array}{c}
\widetilde{\boldsymbol{q}} \\
\dot{\boldsymbol{q}}
\end{array}\right]^{\top}\left[\begin{array}{cc}
\lambda_{\max }(\widetilde{\mathcal{L}}) \boldsymbol{I}_{(n-1) p}+\frac{1}{2} \widetilde{\boldsymbol{T}}_{p}^{\top} \boldsymbol{K} \widetilde{\boldsymbol{T}}_{p} & \frac{1}{2} \mu^{-1}\left(\bar{\gamma} k_{\bar{M}}+\delta\right) \widetilde{\boldsymbol{T}}_{p}^{\top} \\
\frac{1}{2} \mu^{-1}\left(\bar{\gamma} k_{\bar{M}}+\delta\right) \widetilde{\boldsymbol{T}}_{p} & \frac{1}{2}\left(\bar{\gamma} k_{\bar{M}}+\delta\right) \boldsymbol{I}_{n p}
\end{array}\right]\left[\begin{array}{c}
\widetilde{\boldsymbol{q}} \\
\dot{\boldsymbol{q}}
\end{array}\right]
$$

The definitions of $\widetilde{\boldsymbol{T}}_{p}$ and $\widetilde{\boldsymbol{q}}$ are given in Section 2.2.2. The scalar $\delta>0$ is fixed, and will defined further below. Note that $\left(k_{\bar{M}}+\delta\right) \boldsymbol{I}_{n p}>\boldsymbol{M}$ and that $\bar{V}_{\mu}$ is not a Lyapunov function; it is a Lyapunov-like function to be used for stability analysis in the sequel. Let the matrix in (24) be $\boldsymbol{G}$, and it follows that $\bar{V}_{\mu}$ is positive definite in $\widetilde{\boldsymbol{q}}, \dot{\boldsymbol{q}}$ if $\boldsymbol{G}>0$. Theorem 1 and Lemma 4 (specifically expression (14a)) are used to conclude that $G>0$ for all $\mu \geq \mu_{1}^{*}$ such that

$$
\mu_{1}^{*}>\sqrt{\frac{\bar{\gamma} k_{\bar{M}}+\delta}{2 \lambda_{\max }(\widetilde{\mathcal{L}})+\lambda_{\min }(\boldsymbol{K})}}
$$

While $\bar{V}_{\mu}$ is a function of $\widetilde{\boldsymbol{q}}$ and $\dot{\boldsymbol{q}}$, we use $\bar{V}_{\mu}(t)$ to denote $\bar{V}_{\mu}(\widetilde{\boldsymbol{q}}(t), \dot{\boldsymbol{q}}(t))$. Property P1 is used to yield

$$
\bar{V}_{\mu} \leq\left(\frac{1}{2} \lambda_{\max }(\boldsymbol{K})+\lambda_{\max }(\widetilde{\mathcal{L}})\right)\|\widetilde{\boldsymbol{q}}\|_{2}{ }^{2}+\frac{1}{2}\left(\bar{\gamma} k_{\bar{M}}+\delta\right)\|\dot{\boldsymbol{q}}\|_{2}{ }^{2}+\mu^{-1}\left(\bar{\gamma} k_{\bar{M}}+\delta\right)\|\widetilde{\boldsymbol{q}}\|_{2}\|\dot{\boldsymbol{q}}\|_{2}
$$

Next, let

$$
\widehat{V}_{\mu}=\left[\begin{array}{c}
\widetilde{\boldsymbol{q}} \\
\dot{\boldsymbol{q}}
\end{array}\right]^{\top}\left[\begin{array}{cc}
\frac{1}{4} \lambda_{2}(\widetilde{\mathcal{L}}) \boldsymbol{I}_{(n-1) p}+\frac{1}{2} \widetilde{\boldsymbol{T}}_{p}^{\top} \boldsymbol{K} \widetilde{\boldsymbol{T}}_{p} & \frac{1}{2} \mu^{-1}\left(\underline{\gamma} k_{\underline{m}}-\delta\right) \widetilde{\boldsymbol{T}}_{p}^{\top} \\
\frac{1}{2} \mu^{-1}\left(\underline{\gamma} k_{\underline{m}}-\delta\right) \widetilde{\boldsymbol{T}}_{p} & \frac{1}{2}\left(\underline{\gamma k_{m}}-\delta\right) \boldsymbol{I}_{n p}
\end{array}\right]\left[\begin{array}{c}
\widetilde{\boldsymbol{q}} \\
\dot{\boldsymbol{q}}
\end{array}\right]
$$

where $\underline{\gamma}$ is the smallest diagonal element of $\boldsymbol{\Gamma}$ defined in Lemma 3 and is necessarily less than 1 . Call the matrix in (27) $\boldsymbol{L}$. Let $\delta$ be further constrained to require that $\left(\underline{\gamma} k_{\underline{m}}-\delta\right)>0$ holds. From 
Theorem 1 and expression (15), one can conclude that $\boldsymbol{L}>0$ for all $\mu \geq \mu_{2}^{*}$ such that

$$
\mu_{2}^{*}>\sqrt{\frac{2\left(\underline{\gamma} k_{\underline{m}}-\delta\right)}{\lambda_{2}(\widetilde{\mathcal{L}})+2 \lambda_{\min }(\boldsymbol{K})}}
$$

and because $\lambda_{2}(\widetilde{\mathcal{L}})>0$, there always exists a $\mu$ satisfying the inequality. Set $\mu_{3}^{*}=\max \left\{\mu_{1}^{*}, \mu_{2}^{*}\right\}$. Define

$$
\begin{aligned}
& \rho_{1}(\mu)=\frac{1}{2}\left(\bar{\gamma} k_{\bar{M}}+\delta\right)-\frac{1}{4} \mu^{-2}\left(\bar{\gamma} k_{\bar{M}}+\delta\right)^{2} \lambda_{\max }(\widetilde{\mathcal{L}})^{-1} \\
& \rho_{2}(\mu)=\frac{1}{2}\left(\underline{\gamma} k_{\underline{m}}-\delta\right)-\mu^{-2}\left(\underline{\gamma} k_{\underline{m}}-\delta\right)^{2} \lambda_{2}(\widetilde{\mathcal{L}})^{-1} \\
& \rho_{3}(\mu)=\frac{1}{2} \underline{\gamma} k_{\underline{m}}-\frac{1}{2} \mu^{-2}\left(\bar{\gamma} k_{\bar{M}}\right)^{2} \lambda_{2}(\widetilde{\mathcal{L}})^{-1}
\end{aligned}
$$

and observe that for sufficiently large $\mu$ there holds $\rho_{1}(\mu)>\rho_{2}(\mu)$ and $\rho_{3}(\mu)>\rho_{2}(\mu)$. Assume without loss of generality that both $\rho_{1}(\mu)>\rho_{2}(\mu)$ and $\rho_{3}(\mu)>\rho_{2}(\mu)$ hold (if not, one could always find a $\mu_{4}^{*}>\mu_{3}^{*}$ such that $\rho_{1}(\mu)>\rho_{2}(\mu)$ and $\rho_{3}(\mu)>\rho_{2}(\mu)$ for all $\left.\mu \geq \mu_{4}^{*}\right)$. Recalling that $\|\widetilde{\boldsymbol{q}}\|_{2} \leq\|\boldsymbol{q}(0)\|_{2} \leq k_{a}$ and $\|\dot{\boldsymbol{q}}(0)\|_{2} \leq k_{b}$, compute

$$
\bar{V}^{*}=\left(\frac{1}{2} \lambda_{\max }(\boldsymbol{K})+\lambda_{\max }(\widetilde{\mathcal{L}})\right) k_{a}{ }^{2}+\frac{1}{2}\left(\bar{\gamma} k_{\bar{M}}+\delta\right) k_{b}{ }^{2}+\left(\mu_{3}^{*}\right)^{-1}\left(\bar{\gamma} k_{\bar{M}}+\delta\right) k_{a} k_{b}
$$

and note that for any $\mu \geq \mu_{3}^{*}$ there holds $\bar{V}_{\mu}(0) \leq \bar{V}^{*}$ and $\rho_{1}\left(\mu_{3}^{*}\right) \leq \rho_{1}(\mu)$. Furthermore, we have assumed that $\rho_{1}(\mu)>\rho_{2}(\mu)$. It follows from Lemma 1 and (5) that

$$
\|\dot{\boldsymbol{q}}(0)\|_{2} \leq \sqrt{\frac{\bar{V}_{\mu}(0)}{\rho_{1}(\mu)}} \leq \sqrt{\frac{\bar{V}_{\mu}(0)}{\rho_{1}\left(\mu_{3}^{*}\right)}} \leq \sqrt{\frac{\bar{V}^{*}}{\rho_{1}\left(\mu_{3}^{*}\right)}}<\sqrt{\frac{\bar{V}^{*}}{\rho_{2}\left(\mu_{3}^{*}\right)}}:=\mathcal{Y}
$$

Following the same process, the bound $\|\widetilde{\boldsymbol{q}}(0)\|_{2}<\mathcal{X}$ can also be obtained using $\bar{V}^{*}$ and $\rho_{2}\left(\mu_{3}^{*}\right)$. Note that the quantity $\mathcal{Y}$ in (31) is independent of $\mu$. The bound $\|\dot{\boldsymbol{q}}(0)\|_{2}<\mathcal{Y}$ (and similarly $\left.\|\widetilde{\boldsymbol{q}}(0)\|_{2}<\mathcal{X}\right)$ can therefore be used for all $\mu \geq \mu_{3}^{*}$. We now proceed to the main result.

\subsection{Stability Proof}

\section{Theorem 3}

Suppose each Euler-Lagrange agent, with dynamics described by (19), uses the model-independent control law, (20). Then the consensus objective is semi-globally achieved at an exponential rate if $\mathcal{G}$ is strongly connected, and if $\mu$ exceeds lower bounds computable from eigenvalue bounds on the matrices in the agent models, bounds on the eigenvalues of $\mathcal{L}$ and the bounds on the initial conditions $k_{a}, k_{b}$. For a given strongly connected $\mathcal{G}$, there always exists a $\mu$ which satisfies the lower bounds.

\section{Proof}

The proof will be presented in four parts. In Part 1, we present a Lyapunov-like candidate function, $V$. As will be apparent below, $V$ is not a Lyapunov function because its arguments are not the states of (23). However, analysis of $V$ and its time derivative will show leaderless consensus is achieved. In Part 2, we analyse the derivative $\dot{V}$ and conclude a negative definite property. Part 3 studies the assumption that the above computed values $\mathcal{X}, \mathcal{Y}$ are such that $\|\widetilde{\boldsymbol{q}}(t)\|_{2}<\mathcal{X}$ and $\|\dot{\boldsymbol{q}}(t)\|_{2}<\mathcal{Y}$ hold for some time interval $\left[0, T_{2}\right)$. We show that a finite $T_{2}$ creates a contradiction, i.e. $T_{2}$ can be taken to be infinite. With $T_{2}$ infinite, we conclude that the trajectories remain bounded for all time. Part 4 concludes the leaderless consensus objective is achieved exponentially fast.

Part 1: Consider the following Lyapunov-like candidate function

$$
V=\frac{1}{2} \widetilde{\boldsymbol{q}}^{\top} \widetilde{\boldsymbol{T}}_{p}^{\top}\left(\left(\widetilde{\mathcal{L}} \otimes \boldsymbol{I}_{p}\right)+\boldsymbol{K}\right) \widetilde{\boldsymbol{T}}_{p} \widetilde{\boldsymbol{q}}+\mu^{-1} \widetilde{\boldsymbol{q}}^{\top} \widetilde{\boldsymbol{T}}_{p}^{\top} \boldsymbol{\Gamma}_{p} \boldsymbol{M} \dot{\boldsymbol{q}}+\frac{1}{2} \dot{\boldsymbol{q}}^{\top} \boldsymbol{\Gamma}_{p} \boldsymbol{M} \dot{\boldsymbol{q}}=V_{1}+V_{2}+V_{3}
$$


where $\boldsymbol{\Gamma}_{p}=\boldsymbol{\Gamma} \otimes \boldsymbol{I}_{p}$, and $\widetilde{\mathcal{L}}, \boldsymbol{\Gamma}, \widetilde{\boldsymbol{q}}$ and $\widetilde{\boldsymbol{T}}_{p}$ were given below (24). Note that $\boldsymbol{\Gamma}_{p} \boldsymbol{M}$ is symmetric. In quadratic form, $V$ is expressed as

$$
V=\left[\begin{array}{c}
\widetilde{\boldsymbol{q}} \\
\dot{\boldsymbol{q}}
\end{array}\right]^{\top}\left[\begin{array}{cc}
\frac{1}{2} \widetilde{\boldsymbol{T}}_{p}^{\top}\left(\left(\widetilde{\mathcal{L}} \otimes \boldsymbol{I}_{p}\right)+\boldsymbol{K}\right) \widetilde{\boldsymbol{T}}_{p} & \frac{1}{2} \mu^{-1} \widetilde{\boldsymbol{T}}_{p}^{\top} \boldsymbol{\Gamma}_{p} \boldsymbol{M} \\
\frac{1}{2} \mu^{-1} \boldsymbol{M} \boldsymbol{\Gamma}_{p} \widetilde{\boldsymbol{T}}_{p} & \frac{1}{2} \boldsymbol{\Gamma}_{p} \boldsymbol{M}
\end{array}\right]\left[\begin{array}{c}
\widetilde{\boldsymbol{q}} \\
\dot{\boldsymbol{q}}
\end{array}\right]
$$

Define the matrix in (33) as $\boldsymbol{H}$. It follows that $V$ is positive definite in the variables $\widetilde{\boldsymbol{q}}, \dot{\boldsymbol{q}}$ if $\boldsymbol{H}>0$, and notice that $\boldsymbol{H}$ depends on $\boldsymbol{q}$. From Theorem 1 and the assumed properties of $\boldsymbol{M}_{i}$, it follows that $\boldsymbol{H}>0$ if and only if

$$
\frac{1}{2} \widetilde{\boldsymbol{T}}_{p}^{\top}\left(\left(\widetilde{\mathcal{L}} \otimes \boldsymbol{I}_{p}\right)+\boldsymbol{K}\right) \widetilde{\boldsymbol{T}}_{p}-\frac{1}{2} \mu^{-2} \widetilde{\boldsymbol{T}}_{p}^{\top} \boldsymbol{\Gamma}_{p} \boldsymbol{M} \widetilde{\boldsymbol{T}}_{p}>0
$$

Lemma 4 and the properties of the spectral norm yield $\lambda_{\max }\left(\widetilde{\boldsymbol{T}}_{p}^{\top} \boldsymbol{\Gamma}_{p} \boldsymbol{M} \widetilde{\boldsymbol{T}}_{p}\right) \leq \lambda_{\max }\left(\boldsymbol{\Gamma}_{p} \boldsymbol{M}\right)<\bar{\gamma} k_{\bar{M}}$. Corollary 1 and the properties of the Kronecker product yield $\lambda_{\min }\left(\widetilde{\boldsymbol{T}}_{p}^{\top}\left(\widetilde{\mathcal{L}} \otimes \boldsymbol{I}_{p}\right) \widetilde{\boldsymbol{T}}_{p}\right)=\lambda_{2}(\widetilde{\mathcal{L}})$. Combining this with (1), it follows that the inequality (34) is implied by

$$
\mu>\sqrt{\frac{\bar{\gamma} k_{\bar{M}}}{\lambda_{2}(\widetilde{\mathcal{L}})+\lambda_{\min }(\boldsymbol{K})}}
$$

and there always exists such a $\mu$. Although $V$ is a function of $\widetilde{\boldsymbol{q}}, \dot{\boldsymbol{q}}$, for simplicity we denote $V(\widetilde{\boldsymbol{q}}(t), \dot{\boldsymbol{q}}(t))$ as $V(t)$. It is straightforward to verify for all $\mu \geq \mu_{5}^{*}$ such that

$$
\mu_{5}^{*}>\sqrt{\frac{2 \bar{\gamma} k_{\bar{M}}}{\lambda_{2}(\widetilde{\mathcal{L}})+2 \lambda_{\min }(\boldsymbol{K})}}
$$

there holds $\boldsymbol{G}>\boldsymbol{H}>\boldsymbol{L}>0$ and thus $V(t)<\bar{V}_{\mu}(t), \forall t$ (see Appendix). Since $\boldsymbol{H}>0$ for any $\mu \geq \mu_{5}^{*}$, it follows that $V$ is positive definite in $\widetilde{\boldsymbol{q}}, \dot{\boldsymbol{q}}$. Notice that $\lambda_{\min }(\boldsymbol{H})>\lambda_{\min }(\boldsymbol{L})>0$ and $\lambda_{\max }(\boldsymbol{H})<\lambda_{\max }(\boldsymbol{G})<\infty$. In addition, $\lambda_{\min }(\boldsymbol{L}), \lambda_{\max }(\boldsymbol{G})$ are independent of $\boldsymbol{q}, \dot{\boldsymbol{q}}$. It follows that $0<\lambda_{i}(\boldsymbol{H})<\infty, \forall i$ and for all $\widetilde{\boldsymbol{q}}, \dot{\boldsymbol{q}}$. We conclude that $V$ is radially unbounded. From the conclusions obtained immediately above, and in similar fashion to (26), observe that

$$
V \leq \frac{1}{2}\left(\lambda_{\max }(\widetilde{\mathcal{L}})+\lambda_{\max }(\boldsymbol{K})\right)\|\widetilde{\boldsymbol{q}}\|_{2}{ }^{2}+\frac{1}{2} \bar{\gamma} k_{\bar{M}}\|\dot{\boldsymbol{q}}\|_{2}{ }^{2}+\mu^{-1} \bar{\gamma} k_{\bar{M}}\|\widetilde{\boldsymbol{q}}\|_{2}\|\dot{\boldsymbol{q}}\|_{2}
$$

which implies that $V(0)<\bar{V}^{*}$ for all $\mu \geq \mu_{5}^{*}$ because there holds $\mu_{5}^{*} \geq \mu_{3}^{*}$ (as is easily checked from (25), (28) and (36)).

Part 2: The derivative of $V$ with respect to time, along the trajectories of the system (22), yields $\dot{V}=\dot{V}_{1}+\dot{V}_{2}+\dot{V}_{3}$ with notation as in (32). Recalling the properties of $\widetilde{\boldsymbol{T}}_{p}, \boldsymbol{Z}_{p}$ and $\widetilde{\boldsymbol{q}}$ in Sections 2.2.1 and 2.2.2, it follows that

$$
\begin{aligned}
\dot{V}_{1} & =\widetilde{\boldsymbol{q}}^{\top} \widetilde{\boldsymbol{T}}_{p}^{\top}\left(\left(\widetilde{\mathcal{L}} \otimes \boldsymbol{I}_{p}\right)+\boldsymbol{K}\right) \widetilde{\boldsymbol{T}}_{p} \dot{\dot{\boldsymbol{q}}}=\widetilde{\boldsymbol{q}}^{\top} \widetilde{\boldsymbol{T}}_{p}^{\top}\left(\left(\widetilde{\mathcal{L}} \otimes \boldsymbol{I}_{p}\right)+\boldsymbol{K}\right) \boldsymbol{Z}_{p} \dot{\boldsymbol{q}} \\
& =\widetilde{\boldsymbol{q}}^{\top} \widetilde{\boldsymbol{T}}_{p}^{\top}\left(\widetilde{\mathcal{L}} \otimes \boldsymbol{I}_{p}\right) \dot{\boldsymbol{q}}+\widetilde{\boldsymbol{q}}^{\top} \widetilde{\boldsymbol{T}}_{p}^{\top} \boldsymbol{K} \dot{\boldsymbol{q}}
\end{aligned}
$$

with the last term obtained by noting that $K \boldsymbol{Z}_{p}=\boldsymbol{Z}_{p} \boldsymbol{K}$. The derivative $\dot{V}_{2}$ is

$$
\dot{V}_{2}=\mu^{-1} \dot{\tilde{\boldsymbol{q}}}^{\top} \widetilde{\boldsymbol{T}}_{p}^{\top} \boldsymbol{\Gamma}_{p} \boldsymbol{M} \dot{\boldsymbol{q}}+\mu^{-1} \widetilde{\boldsymbol{q}}^{\top} \widetilde{\boldsymbol{T}}_{p}^{\top} \boldsymbol{\Gamma}_{p} \dot{\boldsymbol{M}} \dot{\boldsymbol{q}}+\mu^{-1} \widetilde{\boldsymbol{q}}^{\top} \widetilde{\boldsymbol{T}}_{p}^{\top} \boldsymbol{\Gamma}_{p} \boldsymbol{M} \ddot{\boldsymbol{q}}
$$

Using the properties of the Kronecker product and the properties of $\boldsymbol{Z}_{p}, \widetilde{\boldsymbol{T}}_{p}$, observe that

$$
\boldsymbol{\Gamma}_{p}\left(\mathcal{L} \otimes \boldsymbol{I}_{p}\right) \boldsymbol{q}=\boldsymbol{\Gamma}_{p}\left(\mathcal{L} \otimes \boldsymbol{I}_{p}\right) \boldsymbol{Z}_{p} \boldsymbol{q}=\left(\boldsymbol{\Gamma} \mathcal{L} \otimes \boldsymbol{I}_{p}\right) \widetilde{\boldsymbol{T}}_{p} \widetilde{\boldsymbol{q}}
$$


In addition, by recalling that $\boldsymbol{Z}_{p} \boldsymbol{q}=\widetilde{\boldsymbol{T}}_{p} \widetilde{\boldsymbol{q}}$, substituting $\boldsymbol{M} \ddot{\boldsymbol{q}}$ from (22) and recalling assumption A3, we obtain

$$
\dot{V}_{2}=\mu^{-1} \dot{\boldsymbol{q}}^{\top} \boldsymbol{Z}_{p} \boldsymbol{\Gamma}_{p} \boldsymbol{M} \dot{\boldsymbol{q}}+\mu^{-1} \widetilde{\boldsymbol{q}}^{\top} \widetilde{\boldsymbol{T}}_{p}^{\top} \boldsymbol{\Gamma}_{p} \boldsymbol{C}^{\top} \dot{\boldsymbol{q}}-\mu^{-1} \widetilde{\boldsymbol{q}}^{\top} \widetilde{\boldsymbol{T}}_{p}^{\top}\left(\boldsymbol{\Gamma} \mathcal{L} \otimes \boldsymbol{I}_{p}\right) \widetilde{\boldsymbol{T}}_{p} \widetilde{\boldsymbol{q}}-\widetilde{\boldsymbol{q}}^{\top} \widetilde{\boldsymbol{T}}_{p}^{\top} \boldsymbol{K} \dot{\boldsymbol{q}}
$$

Lastly, $\dot{V}_{3}=\dot{\boldsymbol{q}}^{\top} \boldsymbol{\Gamma}_{p} \boldsymbol{M} \ddot{\boldsymbol{q}}+\frac{1}{2} \dot{\boldsymbol{q}}^{\top} \boldsymbol{\Gamma}_{p} \dot{\boldsymbol{M}} \dot{\boldsymbol{q}}$. Analysis similar to that of $\dot{V}_{2}$ above yields

$$
\dot{V}_{3}=-\dot{\boldsymbol{q}}^{\top}\left(\boldsymbol{\Gamma} \mathcal{L} \otimes \boldsymbol{I}_{p}\right) \widetilde{\boldsymbol{T}}_{p} \widetilde{\boldsymbol{q}}-\mu \dot{\boldsymbol{q}}^{\top} \boldsymbol{K} \dot{\boldsymbol{q}}
$$

Combining $\dot{V}_{1}+\dot{V}_{2}+\dot{V}_{3}$, and recognising that the symmetric part of $\boldsymbol{\Gamma} \mathcal{L}$ is $\frac{1}{2} \widetilde{\mathcal{L}}$, we obtain

$$
\begin{aligned}
\dot{V}=- & {\left[\mu^{-1} \frac{1}{2} \widetilde{\boldsymbol{q}}^{\top} \widetilde{\boldsymbol{T}}_{p}^{\top}\left(\widetilde{\mathcal{L}} \otimes \boldsymbol{I}_{p}\right) \widetilde{\boldsymbol{T}}_{p} \widetilde{\boldsymbol{q}}+\mu \dot{\boldsymbol{q}}^{\top} \boldsymbol{K} \dot{\boldsymbol{q}}-\mu^{-1} \dot{\boldsymbol{q}}^{\top} \boldsymbol{Z}_{p} \boldsymbol{\Gamma}_{p} \boldsymbol{M} \dot{\boldsymbol{q}}-\mu^{-1} \widetilde{\boldsymbol{q}}^{\top} \widetilde{\boldsymbol{T}}_{p}^{\top} \boldsymbol{\Gamma}_{p} \boldsymbol{C}^{\top} \dot{\boldsymbol{q}}\right.} \\
& \left.-\widetilde{\boldsymbol{q}}^{\top} \widetilde{\boldsymbol{T}}_{p}^{\top}\left(\boldsymbol{\Gamma} \mathcal{L} \otimes \boldsymbol{I}_{p}\right) \dot{\boldsymbol{q}}\right]
\end{aligned}
$$

Note that (42) can be written in quadratic form, similar to (33) but there is a difficulty with using the form: assumption $\mathrm{A} 4$, along with the property of norms, yields $\left\|\boldsymbol{C}^{\top}\right\|_{2} \leq k_{C}\|\dot{\boldsymbol{q}}\|_{2}$. The fact that $\|\boldsymbol{C}\|_{2}$ is proportional to $\|\dot{\boldsymbol{q}}\|_{2}$ makes it difficult to apply Theorem 1 to obtain a definiteness property for the block matrix in the quadratic form for $\dot{V}$. This motivates us to consider the following alternative method for studying the definiteness of $\dot{V}$.

From the fact that $\|\boldsymbol{\Gamma}\|_{2}<1$, and with property P1, we obtain

$$
\widetilde{\boldsymbol{q}}^{\top} \widetilde{\boldsymbol{T}}_{p}^{\top} \boldsymbol{\Gamma}_{p} \boldsymbol{C}^{\top} \dot{\boldsymbol{q}} \leq k_{C} \bar{\gamma}\|\widetilde{\boldsymbol{q}}\|_{2}\|\dot{\boldsymbol{q}}\|_{2}^{2}
$$

Using spectral norm properties, assumption A2, Corollary 1 and property P1 it follows that

$$
\begin{aligned}
-\dot{V} & \geq\left(\mu \lambda_{\min }(\boldsymbol{K})-\mu^{-1} \bar{\gamma} k_{\bar{M}}\right)\|\dot{\boldsymbol{q}}\|_{2}{ }^{2}+\frac{1}{2} \mu^{-1} \lambda_{2}(\widetilde{\mathcal{L}})\|\widetilde{\boldsymbol{q}}\|_{2}{ }^{2}-\mu^{-1} k_{C} \bar{\gamma}\|\widetilde{\boldsymbol{q}}\|_{2}\|\dot{\boldsymbol{q}}\|_{2}{ }^{2} \\
& -\|\mathcal{L}\|_{2}\|\widetilde{\boldsymbol{q}}\|_{2}\|\dot{\boldsymbol{q}}\|_{2}:=g\left(\|\widetilde{\boldsymbol{q}}\|_{2},\|\dot{\boldsymbol{q}}\|_{2}\right)
\end{aligned}
$$

Define the region given by $\|\widetilde{\boldsymbol{q}}(t)\|_{2} \in[0, \infty)$ and $\|\dot{\boldsymbol{q}}(t)\|_{2} \in[0, \mathcal{Y}]$ as $\mathcal{D}$, where $\mathcal{Y}>0$ was computed in Section 3.1. Observe that $g\left(\|\widetilde{\boldsymbol{q}}\|_{2},\|\dot{\boldsymbol{q}}\|_{2}\right)$ in (44) is of the form of $g(x, y)$ given in Lemma 2 with $\|\widetilde{\boldsymbol{q}}\|_{2}=x$ and $\|\dot{\boldsymbol{q}}\|_{2}=y$, with $\frac{1}{2} \mu^{-1} \lambda_{2}(\widetilde{\mathcal{L}})=a, \mu \gamma \lambda_{\min }(\boldsymbol{K})-\mu^{-1} \bar{\gamma} k_{\bar{M}}=b, \mu^{-1} k_{C} \bar{\gamma}=c$ and $\|\mathcal{L}\|_{2}=d$. According to Lemma 2 , designing $\mu$ such that

$$
\mu \lambda_{\min }(\boldsymbol{K})-\mu^{-1} \bar{\gamma} k_{\bar{M}}>\frac{\left(\mu^{-1} k_{C} \bar{\gamma}\right)^{2} \mathcal{Y}^{2}+\|\mathcal{L}\|_{2}^{2}+2 \mu^{-1} k_{C} \bar{\gamma}\|\mathcal{L}\|_{2} \mathcal{Y}}{2 \mu^{-1} \lambda_{2}(\widetilde{\mathcal{L}})}
$$

ensures $\dot{V} \leq-g\left(\|\widetilde{\boldsymbol{q}}\|_{2},\|\dot{\boldsymbol{q}}\|_{2}\right)<0$ for all $\|\widetilde{\boldsymbol{q}}\|_{2},\|\dot{\boldsymbol{q}}\|_{2} \in \mathcal{D}$. Straightforward calculations show that (45) is implied by

$$
\mu^{2}\left(2 \lambda_{\min }(\boldsymbol{K}) \lambda_{2}(\widetilde{\mathcal{L}})-\|\mathcal{L}\|_{2}{ }^{2}\right)-2 \mu k_{C} \bar{\gamma}\|\mathcal{L}\|_{2} \mathcal{Y}-\left(\left(k_{C} \bar{\gamma} \mathcal{Y}\right)^{2}+2 \lambda_{2}(\widetilde{\mathcal{L}}) \bar{\gamma} k_{\bar{M}}\right)>0
$$

Because $\boldsymbol{K}_{p}$ satisfies (21), the coefficient of $\mu^{2}$ in (46) is strictly positive. Thus, one can always find a $\mu_{6}^{*} \geq \mu_{5}^{*}$ such that (46) holds for all $\mu \geq \mu_{6}^{*}$; this ensures that $\dot{V}<0$ in the region $\mathcal{D}$.

Part 3: With the objective of obtaining a contradiction, suppose that $T_{2}<\infty$ is the infimum of those values of $t$ for which one of the inequalities $\|\widetilde{\boldsymbol{q}}(t)\|_{2}<\mathcal{X},\|\dot{\boldsymbol{q}}(t)\|_{2}<\mathcal{Y}$ fails to hold. In other words, $\left[0, T_{2}\right)$ is maximal. Note that $T_{2}$ is necessarily strictly positive, since the values of $\widetilde{\boldsymbol{q}}(t), \dot{\boldsymbol{q}}(t)$ necessarily change continuously. The strict inequalities are satisfied at $t=0$. Define the compact region $\mathcal{W} \subset \mathcal{D}$ as

$$
\mathcal{W}:=\left\{\widetilde{\boldsymbol{q}}, \dot{\boldsymbol{q}}:\|\widetilde{\boldsymbol{q}}\|_{2} \in[0, \mathcal{X}],\|\dot{\boldsymbol{q}}\|_{2} \in[0, \mathcal{Y}]\right\}
$$

It is obvious that at $t=0$, the system is in $\mathcal{W}$ and thus $\dot{V}<0$. In fact, $\dot{V}<0$ will continue to hold up to and including $t=T_{2}$. This implies that $V\left(T_{2}\right)<V(0)$. In addition, recall the conclusion below 
(37) that $V(0)<\bar{V}^{*}$, where $\bar{V}^{*}$ was computed in Section 3.1. Shortly, we use Lemma 1 on the matrix $\boldsymbol{H}$ in (33). In anticipation of this, let

$$
\chi=\lambda_{\min }\left(\frac{1}{2} \boldsymbol{\Gamma}_{p} \boldsymbol{M}-\frac{1}{2} \mu^{-2} \boldsymbol{\Xi}\right)
$$

where $\boldsymbol{\Xi}=\boldsymbol{M} \boldsymbol{\Gamma}_{p} \widetilde{\boldsymbol{T}}_{p}\left(\widetilde{\boldsymbol{T}}_{p}^{\top}\left(\widetilde{\mathcal{L}} \otimes \boldsymbol{I}_{p}\right) \widetilde{\boldsymbol{T}}_{p}\right)^{-1} \widetilde{\boldsymbol{T}}_{p}^{\top} \boldsymbol{\Gamma}_{p} \boldsymbol{M}$. From (3), we observe that $\chi \geq \lambda_{\min }\left(\frac{1}{2} \boldsymbol{\Gamma}_{p} \boldsymbol{M}\right)-$ $\lambda_{\text {max }}(\boldsymbol{\Xi})$. Recall that $\widetilde{\boldsymbol{T}}_{p}^{\top}\left(\widetilde{\mathcal{L}} \otimes \boldsymbol{I}_{p}\right) \widetilde{\boldsymbol{T}}_{p}>0$. From the fact that $\operatorname{rank}(\boldsymbol{A} \boldsymbol{B}) \leq \min \{\operatorname{rank}(\boldsymbol{A}), \operatorname{rank}(\boldsymbol{B})\}$ [31], we conclude $\operatorname{rank}\left(\boldsymbol{M} \boldsymbol{\Gamma}_{p} \widetilde{\boldsymbol{T}}_{p}\right) \leq(n-1) p$. It follows from these two observations that $\boldsymbol{\Xi} \geq 0$ and $\operatorname{rank}(\boldsymbol{\Xi}) \leq(n-1) p$, which implies that $\lambda_{\max }(\boldsymbol{\Xi})=\|\boldsymbol{\Xi}\|_{2}$. It is straightforward to compute, using Lemma 4, that $\|\boldsymbol{\Xi}\|_{2} \geq\left(\bar{\gamma} k_{\bar{M}}\right)^{2} \lambda_{2}(\widetilde{\mathcal{L}})^{-1}$. It follows that

$$
\chi \geq \frac{1}{2} \underline{\gamma} k_{\underline{m}}-\frac{1}{2} \mu^{-2}\left(\bar{\gamma} k_{\bar{M}}\right)^{2} \lambda_{2}(\widetilde{\mathcal{L}})^{-1}=\rho_{3}(\mu)>\rho_{2}(\mu)
$$

In accordance with Lemma 1, it follows that there holds

$$
\left\|\dot{\boldsymbol{q}}\left(T_{2}\right)\right\|_{2} \leq \sqrt{\frac{V\left(T_{2}\right)}{\chi}}<\sqrt{\frac{\bar{V}^{*}}{\chi}}<\sqrt{\frac{\bar{V}^{*}}{\rho_{2}\left(\mu_{3}^{*}\right)}}=\mathcal{Y}
$$

A similar expression to (50) can be obtained to show $\left\|\widetilde{\boldsymbol{q}}\left(T_{2}\right)\right\|_{2}<\mathcal{X}$. The existence of (50) (and similarly $\left.\left\|\widetilde{\boldsymbol{q}}\left(T_{2}\right)\right\|_{2}<\mathcal{X}\right)$ establishes that $T_{2}$ is not maximal, which contradicts the definition of $T_{2}$. As a result, the bounds $\|\widetilde{\boldsymbol{q}}(t)\|_{2}<\mathcal{X},\|\dot{\boldsymbol{q}}(t)\|_{2}<\mathcal{Y}$ hold for all time. See Remark 4 below for further insight into the bounds.

Part 4: Because we have established that the bounds, explored immediately above, hold for all time, then we draw the conclusion that trajectory of the system (22) remains in the region $\mathcal{W} \subset \mathcal{D}$ for all time. Recall the conclusion below (36) that $\boldsymbol{G}>\boldsymbol{H}>\boldsymbol{L}>0$. It follows that

$$
\lambda_{\min }(\boldsymbol{H})\left\|\left[\widetilde{\boldsymbol{q}}^{\top}, \dot{\boldsymbol{q}}^{\top}\right]^{\top}\right\|_{2}^{2} \leq V \leq \lambda_{\max }(\boldsymbol{G})\left\|\left[\widetilde{\boldsymbol{q}}^{\top}, \dot{\boldsymbol{q}}^{\top}\right]^{\top}\right\|_{2}^{2}
$$

Next, observe that $\dot{V}$ is upper bounded by $-g\left(\|\widetilde{\boldsymbol{q}}\|_{2},\|\dot{\boldsymbol{q}}\|_{2}\right)$ and $-g\left(\|\widetilde{\boldsymbol{q}}\|_{2},\|\dot{\boldsymbol{q}}\|_{2}\right)$ is negative definite in the compact region $\mathcal{W}$. Because $\mathcal{W}$ is compact, there exists a strictly positive $\xi$ such that $\dot{V} \leq-g\left(\|\widetilde{\boldsymbol{q}}\|_{2},\|\dot{\boldsymbol{q}}\|_{2}\right) \leq-\xi\left\|\left[\widetilde{\boldsymbol{q}}^{\top}, \dot{\boldsymbol{q}}^{\top}\right]^{\top}\right\|_{2}{ }^{2}<0$ holds in $\mathcal{W}$. It follows that $\dot{V} \leq-\psi V$ where $\psi=$ $\xi / \lambda_{\max }(\boldsymbol{G})$ and the strict positivity of $\xi$ and $\lambda_{\max }(\boldsymbol{G})$ ensures $\psi$ is strictly positive. This allows us to conclude that $V$ decays to zero exponentially fast, with a minimum rate of $e^{-\psi t}$. The inequality $\lambda_{\min }(\boldsymbol{H})\left\|\left[\widetilde{\boldsymbol{q}}^{\top}, \dot{\boldsymbol{q}}^{\top}\right]^{\top}\right\|_{2}{ }^{2} \leq V$ will then imply that $\left[\widetilde{\boldsymbol{q}}^{\top}, \dot{\boldsymbol{q}}^{\top}\right]^{\top}$ decays to zero exponentially fast. We conclude that $\widetilde{\boldsymbol{q}}$ and $\dot{\boldsymbol{q}}$ decay to zero. Recalling that $\widetilde{\boldsymbol{T}} \widetilde{\boldsymbol{q}}=\boldsymbol{Z}_{p} \boldsymbol{q}$, it follows that $\boldsymbol{Z}_{p} \boldsymbol{q}$ decays to zero. Recalling the nullspace of $\boldsymbol{Z}_{p}$, observe that $\boldsymbol{Z}_{p} \boldsymbol{q}=\mathbf{0}$ implies $\boldsymbol{q}=\mathbf{1}_{n} \otimes \boldsymbol{x}$ where $\boldsymbol{x} \in \mathbb{R}^{p}$. Because $\left\{\boldsymbol{q}=\mathbf{1}_{n} \otimes \boldsymbol{x}, \dot{\boldsymbol{q}}=\mathbf{0}\right\} \in \mathcal{M}$, this implies that the system (23) reaches a point in its set of equilibrium points. Convergence must in fact be to a single point in $\mathcal{M}$, because in $\mathcal{M}$ there holds $\dot{\boldsymbol{q}}=\mathbf{0}$. The leaderless consensus objective is achieved and the proof is completed.

We conclude the stability proof section by providing a simple step-by-step guide to designing the control gains $\mu, \boldsymbol{K}_{p}$.

1. Design $\boldsymbol{K}_{p}$ to satisfy (21) and for all following steps, assume $\boldsymbol{K}_{p}$ is fixed.

2. Compute $\mu_{3}^{*}=\max \left\{\mu_{1}^{*}, \mu_{2}^{*}\right\}$ using (25) and (28), then compute $\bar{V}^{*}$ using (30). Next, compute $\mathcal{Y}$ using (31). Lastly, compute $\mu_{5}^{*}$ according to (36).

3. Compute $\mu_{6}^{*} \geq \mu_{5}^{*}$ to satisfy (46) and set $\mu \geq \mu_{6}^{*}$.

Remark 3 (Knowledge of Global Information)

Design of $\mu$ requires limited knowledge of parameters associated with the multiagent system; this 
implies centralised design as opposed to the implementation of control laws. Specifically, the bounds $k_{C}, k_{\underline{m}}, k_{\bar{M}}$, which are associated with the agent dynamic models, are required. In terms of the network topology, $\lambda_{2}(\widetilde{\mathcal{L}}),\|\mathcal{L}\|_{2}, \gamma$ are required. One can also use bounds if exact information is unavailable (in the case of $\gamma$, approximations may be used as the exponential stability property protects against small variations in the system). We also require the upper bounds on the initial conditions $k_{a}, k_{b}$. It is logical to assume these three limited classes of information are available for most multiagent systems. Other papers studying multiagent coordination of Euler-Lagrange networks also require centralised design of control gains. In [23] and [24], $k_{C}, k_{m}, k_{\bar{M}}, \lambda_{\min }(\mathcal{L})$, bounds on the derivatives of the leader trajectory and the initial conditions are required to design a control gain. In [21], to deal with time-delay, a control gain is designed to be sufficiently large and requires knowledge of the upper bound on the time-delay. Using global knowledge to design control gains for coordination of Euler-Lagrange networks is also reported in e.g. [18, 28], and for coordination of directed networks where agents have general nonlinear dynamics described by Lipschitz continuous functions [38, 39].

Semi-global results in consensus literature include [23, 24, 25, 26, 27, 30, 40], and can arise when the agent dynamics are modelled by nonlinear functions which are not globally Lipschitz (Assumption A4 indicates that typical Euler-Lagrange systems do not satisfy this global Lipschitz condition).

Remark 4 (The quantities $\mathcal{X}$ and $\mathcal{Y}$ )

By using the quantity $\mathcal{Y}$ to design $\mu$ according to (46), we ensure that the trajectory of (22) remains in the region $\mathcal{W}$ for all time because $\dot{V}$ is negative definite in $\mathcal{W}$. The leaderless consensus objective is achieved as a consequence. The bound $\|\widetilde{\boldsymbol{q}}(t)\|_{2}<\mathcal{X}, \forall t$ is in fact not required for computation of a $\mu$ which guarantees stability. However, computation of $\mathcal{X}$ does provide a bound on the system trajectory, and can be used to compute the rate of convergence, $\psi$, both of which may be useful in analysis of system performance. The requirement to compute $\mathcal{Y}$ can be removed for the subclass of Euler-Lagrange equations which satisfies $\left\|\boldsymbol{C}_{i}\right\|_{2} \leq k_{\bar{C}}, \forall i$, with $k_{\bar{C}}$ strictly positive. In other words, the norm of $\boldsymbol{C}_{i}, \forall i$ is upper bounded by a constant. In this case, one can obtain a global stability result.

\section{Remark 5 (Convergence speed)}

The focus throughout the proof of Theorem 3 has been to design $\mu$ to be sufficiently large such that it satisfies several lower bounding inequalities. However, making $\mu$ arbitrarily large has a negative impact on the convergence of (22) to the leaderless consensus objective. Observe that $\mu^{-1}$ is in the coefficient of $\|\widetilde{\boldsymbol{q}}\|_{2}^{2}$ in (44); increasing $\mu$ decreases $\psi$, which leads to slower consensus. On the other hand, a designer may choose to increase $\lambda_{2}(\widetilde{\mathcal{L}})$ by multiplying each $a_{i j}$ of (20) by a large, strictly positive constant, call it $\alpha$; this increases the coefficient of $\|\widetilde{\boldsymbol{q}}\|_{2}{ }^{2}$. However, this also leads to an increase in $\|\mathcal{L}\|_{2}$ in (44), which will require a larger $\lambda_{\min }\left(\boldsymbol{K}_{p}\right)$. As a result, $\psi$ can decrease and slow consensus. This implies that in order to maximise rate of convergence, the graph topology (i.e. edges and their weights) must be designed to minimise the ratio $\lambda_{\max }(\mathcal{L}) / \lambda_{2}(\widetilde{\mathcal{L}})$. These observations are confirmed in the simulations in Section 4.

\section{Remark 6 (Robustness)}

The controller proposed in (20) exhibits robustness in two aspects. Firstly, the controller achieves the leaderless consensus objective exponentially fast. The exponential stability property of the networked system provides improved robustness against small perturbations and disturbances when compared to a system which is asymptotically stable, but not exponentially so. Secondly, the information required to design $\mu$ (including computation of $\mathcal{Y}$ ) requires bounds, rather than exact values. Thus, if there is uncertainty in a parameter, e.g. $k_{\bar{M}}$, then a conservative estimate can be made. This provides robustness against uncertainty in modelling of the multiagent system in a) the agent dynamic model $\left(k_{\bar{M}}, k_{\underline{M}}, k_{C}\right)$, b) the network model $\left(\lambda_{2}(\widetilde{\mathcal{L}}), \lambda_{\max }(\mathcal{L}), \gamma\right)$ and c) initial conditions. In contrast, adaptive algorithms utilising the linear parametrisation of (19) can only handle uncertainty involving constant parameters of (19); adaptive algorithms are intrinsically not robust against unmodelled/uncertain dynamics. 


\subsection{Additional Velocity Information For Better Performance}

Consider additional flow of $\dot{\boldsymbol{q}}_{i}-\dot{\boldsymbol{q}}_{j}$ information between neighbouring agents over the same graph $\mathcal{G}$, which models flow of relative generalised coordinate information. The new control law is proposed as

$$
\tau_{i}=-\sum_{j \in \mathcal{N}_{i}} a_{i j}\left(\left(\boldsymbol{q}_{i}-\boldsymbol{q}_{j}\right)+\mu\left(\dot{\boldsymbol{q}}_{i}-\dot{\boldsymbol{q}}_{j}\right)\right)-\mu \gamma_{i}^{-1} \boldsymbol{K}_{p} \dot{\boldsymbol{q}}_{i}
$$

This yields the closed loop system

$$
\boldsymbol{M}(\boldsymbol{q}) \ddot{\boldsymbol{q}}+\boldsymbol{C}(\boldsymbol{q}, \dot{\boldsymbol{q}}) \dot{\boldsymbol{q}}+\left(\mathcal{L} \otimes \boldsymbol{I}_{p}\right)(\boldsymbol{q}+\mu \dot{\boldsymbol{q}})+\mu \boldsymbol{\Gamma}^{-1} \boldsymbol{K} \dot{\boldsymbol{q}}=\mathbf{0}
$$

and the set of equilibrium points for (53) is also given by $\mathcal{M}$. Due to space limitations and because of its similarity with the proof in Theorem 3, we omit the proof of stability for the system (53) but summarise the key steps of the omitted proof. The bounds involving the quantities $\mathcal{X}, \mathcal{Y}$ are computed as in Section 3.1 using the given $\bar{V}_{\mu}$ and $\hat{V}_{\mu}$. The Lyapunov-like function is the same as that proposed in (33). It is straightforward to verify that the function $\dot{V}$ evaluates to

$$
\begin{aligned}
\dot{V}=- & {\left[\mu^{-1} \frac{1}{2} \widetilde{\boldsymbol{q}}^{\top} \widetilde{\boldsymbol{T}}_{p}^{\top}\left(\widetilde{\mathcal{L}} \otimes \boldsymbol{I}_{p}\right) \widetilde{\boldsymbol{T}}_{p} \widetilde{\boldsymbol{q}}+\mu \dot{\boldsymbol{q}}^{\top}\left(\boldsymbol{K}+\frac{1}{2}\left(\widetilde{\mathcal{L}} \otimes \boldsymbol{I}_{p}\right)\right) \dot{\boldsymbol{q}}-\mu^{-1} \dot{\boldsymbol{q}}^{\top} \boldsymbol{Z}_{p} \boldsymbol{\Gamma}_{p} \boldsymbol{M} \dot{\boldsymbol{q}}\right.} \\
& \left.-\mu^{-1} \widetilde{\boldsymbol{q}}^{\top} \widetilde{\boldsymbol{T}}_{p}^{\top} \boldsymbol{\Gamma}_{p} \boldsymbol{C}^{\top} \dot{\boldsymbol{q}}\right]
\end{aligned}
$$

which is upper bounded as follows

$$
\dot{V} \leq\left[\mu\left(\lambda_{\min }(\boldsymbol{K})+\frac{1}{2} \lambda_{2}(\widetilde{\mathcal{L}})\right)-\mu^{-1} \bar{\gamma} k_{\bar{M}}\right]\|\dot{\boldsymbol{q}}\|_{2}{ }^{2}+\frac{1}{2} \mu^{-1} \lambda_{2}(\widetilde{\mathcal{L}})\|\widetilde{\boldsymbol{q}}\|_{2}{ }^{2}-\mu^{-1} k_{C} \bar{\gamma}\|\widetilde{\boldsymbol{q}}\|_{2}\|\dot{\boldsymbol{q}}\|_{2}{ }^{2}
$$

Straightforward conditions then show that the equivalent condition of (46), which ensures $\dot{V}<0$ in $\mathcal{W}$, is given by

$$
\mu^{2}\left(2 \lambda_{\min }(\boldsymbol{K}) \lambda_{2}(\widetilde{\mathcal{L}})+\lambda_{2}(\widetilde{\mathcal{L}})^{2}\right)-\left(k_{C}{ }^{2} \mathcal{Y}^{2}+2 \lambda_{2}(\widetilde{\mathcal{L}}) k_{\bar{M}}\right)>0
$$

Since the coefficient of $\mu^{2}$ is strictly positive, one can always find a $\mu_{7}^{*} \geq \mu_{5}^{*}$ such that the inequality (56) holds for all $\mu \geq \mu_{7}^{*}$ and thus $\dot{V}<0$ in $\mathcal{W}$. The conclusions on boundedness of the trajectories and exponential convergence to the leaderless consensus objective can then be made using the same arguments as in the proof of Theorem 3.

Notice from (54) that the term $\|\mathcal{L}\|_{2}$ has disappeared, and a new term $\frac{1}{2} \mu \dot{\boldsymbol{q}}^{\top}\left(\widetilde{\mathcal{L}} \otimes \boldsymbol{I}_{p}\right) \dot{\boldsymbol{q}}$ is introduced. This implies that $\psi$ has increased; the additional information results in faster consensus. Furthermore, increasing $\lambda_{2}(\widetilde{\mathcal{L}})$ by multiplying each $a_{i j}$ by a large, strictly positive constant $\alpha$ will increase $\psi$ and result in faster consensus. This is in contrast to the discussions in Remark 5 which showed that $\alpha$ slowed consensus. Notice further that there is no longer an inequality requirement on $\boldsymbol{K}_{p}$. The advantages of smoother transient and faster convergence should be weighed against the additional requirement of generalised velocity information flow, with the specific application of the control law in mind.

\section{Remark 7}

Note that in (52) we have assumed both additional pieces of information have been incorporated. It is possible that only additional relative generalised velocity information is available, or that only $\gamma$ is available. In these cases, similar stability proofs will yield similar lower bounding inequalities which $\mu$ must satisfy to guarantee leaderless consensus is achieved. It is straightforward to show that for both cases, there always exists a $\mu$ which satisfies the lower bounding inequalities. 


\section{SIMULATIONS}

A simulation is provided to demonstrate the distributed algorithm (20) on a network of six two-link revolute robot arms. A model is shown in Fig. 1 and the equations of motion are derived in [8], pp. 259-262 (note that our dynamics has $\boldsymbol{g}_{i}=\mathbf{0}, \forall i$ ). The generalised coordinates for agent $i$ are $\boldsymbol{q}_{i}=\left[q_{i}^{(1)}, q_{i}^{(2)}\right]^{\top}$, which are the angles of each link in radians. The equation of motion for the $i^{t h}$ agent is given as

$$
\left[\begin{array}{ll}
M 11 & M 12 \\
M 21 & M 22
\end{array}\right]\left[\begin{array}{l}
\ddot{q}_{i}^{(1)} \\
\ddot{q}_{i}^{(2)}
\end{array}\right]+\left[\begin{array}{ll}
C 11 & C 12 \\
C 21 & C 22
\end{array}\right]\left[\begin{array}{l}
\dot{q}_{i}^{(1)} \\
\dot{q}_{i}^{(2)}
\end{array}\right]=\left[\begin{array}{l}
\tau_{i}^{(1)} \\
\tau_{i}^{(2)}
\end{array}\right]
$$

The model is shown in Fig. 1 and the elements in the $M_{i}, C_{i}$ matrices are given below:

$$
\begin{aligned}
& -M 11=m_{1} l_{c 1}^{2}+m_{2}\left(l_{1}^{2}+l_{c 2}^{2}+2 l_{1} l_{c 2} \cos q_{i}^{(2)}\right)+I_{1}+I_{2} \\
& -M 12=M 21=m_{2}\left(l_{c 2}^{2}+l_{1} l_{c 2} \cos q_{i}^{(2)}\right)+I_{2} \\
& \text { - } M 22=m_{2} l_{c 2}^{2}+I_{2} \\
& \text { - } C 11=-h \dot{q}_{i}^{(2)} \\
& \text { - } C 12=-h\left(\dot{q}_{i}^{(1)}+\dot{q}_{i}^{(2)}\right) \\
& \text { - } C 21=h \dot{q}_{i}^{(1)} \\
& \text { - } C 22=0 \\
& \text { - } h=m_{2} l_{1} l_{c 2} \sin q_{i}^{(2)}
\end{aligned}
$$

The geometric parameters $m_{1}, m_{2}, l_{1}, l_{2}, l_{c 1}, l_{c 2}, I_{1}, I_{2}$ are shown in Table I with SI units of $\mathrm{kg}$ for $m_{1}, m_{2}$ and $\mathrm{m}$ for $l_{1}, l_{2}, l_{c 1}, l_{c 2}$ and $\mathrm{kg} \mathrm{m}^{2}$ for $I_{1}, I_{2}$. The parameters were selected to be representative of robotic manipulators found in industry [9]. The initial conditions of the system are also provided in Table I with units of radians for the generalised coordinates and radians per second for generalised velocity. The directed graph $\mathcal{G}$, shown in Fig 2, has the following Laplacian

$$
\mathcal{L}=\left[\begin{array}{cccccc}
1.5 & 0 & 0 & 0 & 0 & -1.5 \\
-0.8 & 1.8 & -1.0 & 0 & 0 & 0 \\
0 & -1.5 & 3.2 & -1.5 & -0.2 & 0 \\
0 & 0 & -2.0 & 2.0 & 0 & 0 \\
0 & 0 & 0 & 0 & 0.5 & -0.5 \\
0 & -3 & 0 & 0 & -0.5 & 3
\end{array}\right]
$$

with $\gamma=[0.1718,0.3222,0.1895,0.1421,0.0758,0.0985]^{\top}$ and verify that it is strongly connected. Note that the computed $\mu$ is necessarily conservative due to the inequalities used to ensure stability and boundedness of the trajectories. The computed $\mu$ can be further adjusted using standard techniques, e.g. a line search, to improve convergence performance. The control gain pair is $\mu=1, \boldsymbol{K}_{p}=\boldsymbol{I}_{2}, \forall i$.

The results are shown in Figures 3, 4 and 5. Figure 3 shows consensus of the generalised coordinates $q^{(1)}$ and $q^{(2)}$. The generalised velocities, $\dot{q}^{(1)}$ and $\dot{q}^{(2)}$, all tend to zero in Fig. 4. The control torques are shown in Fig. 5. In general, it is not possible to quantitatively determine the final consensus value of the generalised coordinates. Observations from multiple simulations show that the values $a_{i j}, \mu \boldsymbol{K}_{p}$ and the individual agent parameters all have an effect on the final consensus value, which is clearly dependent on the unknown specific values of the model parameters. Recall from Theorem 3 that we require $\mu$ to satisfy lower bounding inequalities for stability. Figure 6 shows the generalised coordinates of a simulation where the parameters are identical to Table I, with the same graph topology and same $\boldsymbol{K}_{p}$. The only change is that we decrease the control scalar to $\mu=0.4$. We see the instability as a result of the smaller $\mu$. 
Table I. Agent parameters used in simulation

\begin{tabular}{ccccccccccccc}
$\bullet$ & $m_{1}$ & $m_{2}$ & $l_{1}$ & $l_{2}$ & $l_{c 1}$ & $l_{c 2}$ & $I_{1}$ & $I_{2}$ & $q_{i}^{(1)}(0)$ & $q_{i}^{(2)}(0)$ & $\dot{q}_{i}^{(1)}(0)$ & $\dot{q}_{i}^{(2)}(0)$ \\
\hline Agent 1 & 6.5 & 2 & 0.3 & 0.25 & 0.1 & 0.05 & 0.1 & 0.02 & $\pi / 7$ & $3 \pi / 4$ & 0.3 & -0.8 \\
\hline Agent 2 & 4 & 2.5 & 0.3 & 0.3 & 0.15 & 0.08 & 0.15 & 0.05 & $3 \pi / 8$ & $\pi / 8$ & 0.6 & -0.6 \\
\hline Agent 3 & 5.2 & 1.8 & 0.25 & 0.25 & 0.25 & 0.08 & 0.1 & 0.02 & $7 \pi / 8$ & $-5 \pi / 8$ & 0.1 & 0.2 \\
\hline Agent 4 & 3 & 3 & 0.35 & 0.2 & 0.12 & 0.1 & 0.13 & 0.02 & $\pi$ & $-\pi / 2$ & 0.2 & 0.3 \\
\hline Agent 5 & 7 & 1.3 & 0.26 & 0.26 & 0.12 & 0.1 & 0.13 & 0.02 & $5 \pi / 8$ & 0 & 0 & 0.6 \\
\hline Agent 6 & 6.5 & 2.2 & 0.2 & 0.25 & 0.1 & 0.09 & 0.15 & 0.08 & $6 \pi / 7$ & $-\pi / 8$ & -0.5 & 0.1 \\
\hline
\end{tabular}

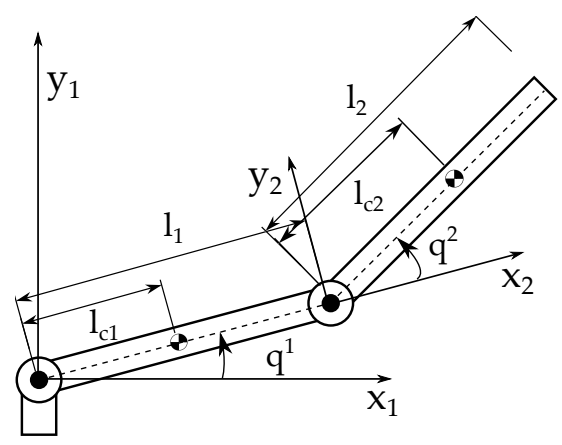

Figure 1. Two-link arm, generalized coordinates

$$
\boldsymbol{q}=\left[q^{1}, q^{2}\right]^{\top}
$$

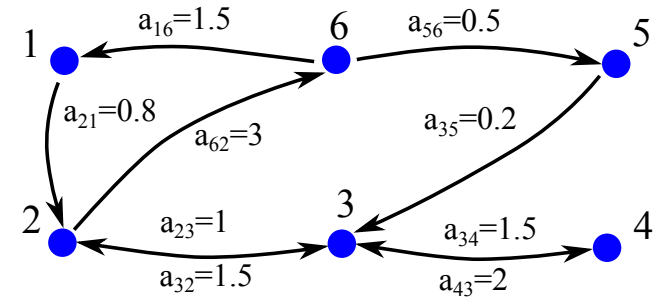

Figure 2. Strongly Connected Graph Used For Simulations
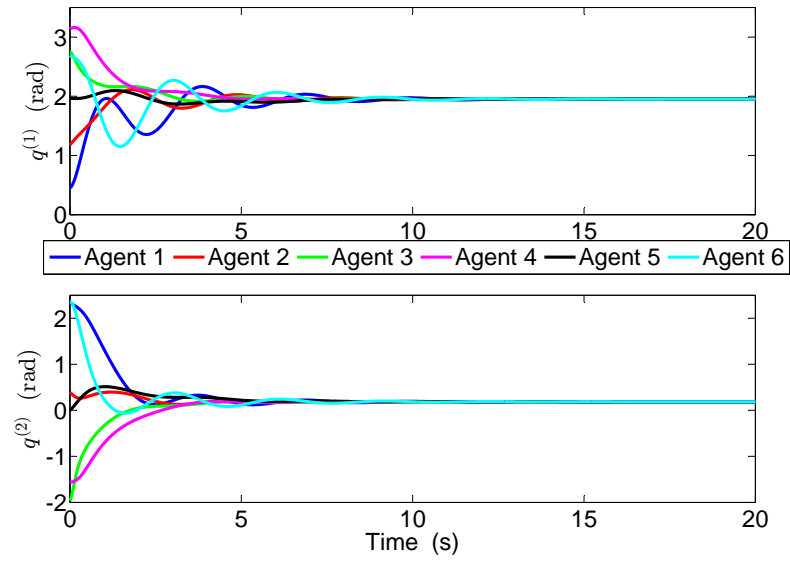

Figure 3. Plot of generalised coordinates vs. time

Conversely, increasing $\mu$ substantially can degrade performance by increasing the time required for consensus. For a simulation where $\mu=5$ and otherwise identical parameters, the results are in Fig. 7. If each $a_{i j}$ is multiplied by 2 with $\mu \boldsymbol{K}_{p}=\boldsymbol{I}_{2}$ then, as observed in Fig. 8, there is an increase in the transient oscillations with slower convergence. Both these results are in accordance with the conclusions in Remark 5. Intuitively, we can consider $\mu \boldsymbol{K}$ as a damping term in the nonlinear system (22); as the damping increases the transient response is slowed. Likewise, the term in (22) involving $\mathcal{L}$ may represent a nonlinear spring in a nonlinear spring-mass-damper system. Lastly, using the algorithm in Section 3.3, Fig. 9 shows fast convergence and smooth transient behaviour when additional information is utilised. 

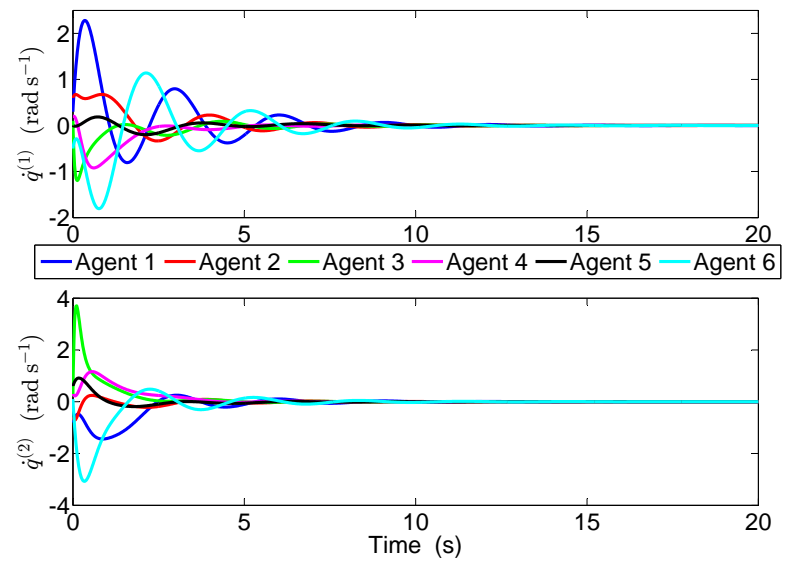

Figure 4. Plot of generalised velocity vs. time

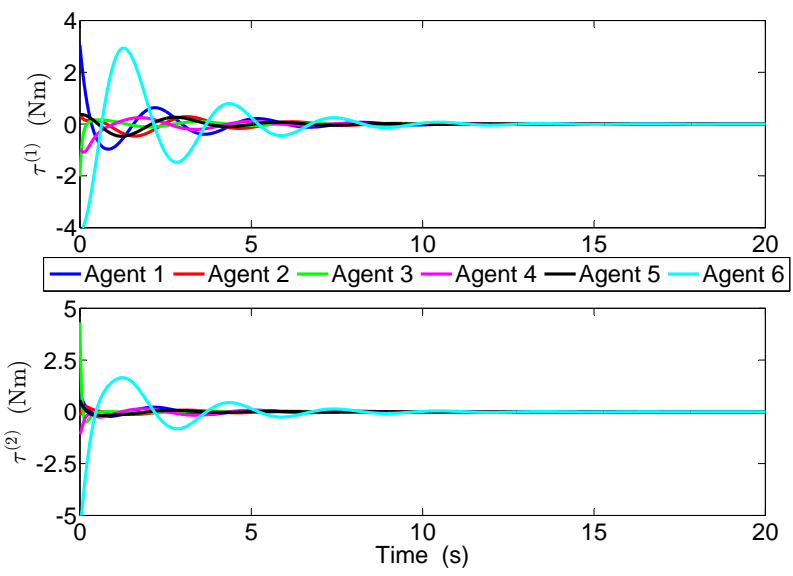

Figure 5. Plot of control input torque vs. time
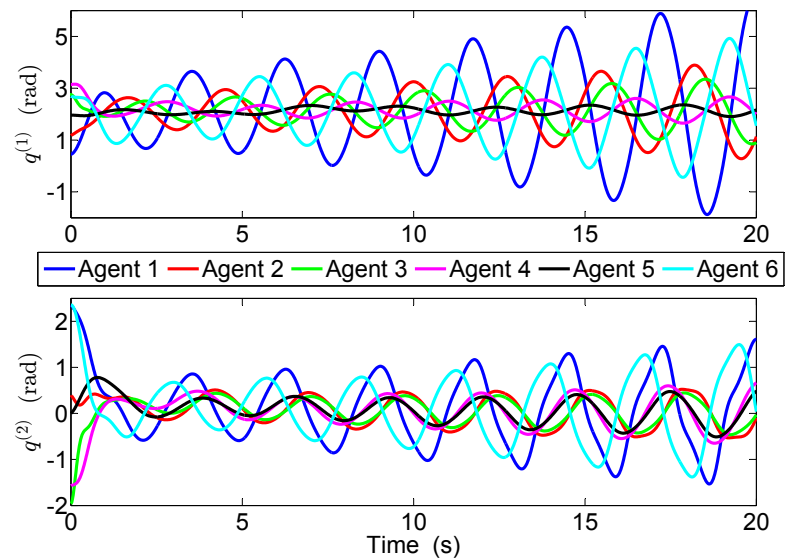

Figure 6. Plot of generalised coordinates vs. time, with unstable $\mu \boldsymbol{K}_{i}=0.4 \boldsymbol{I}_{2}$ 

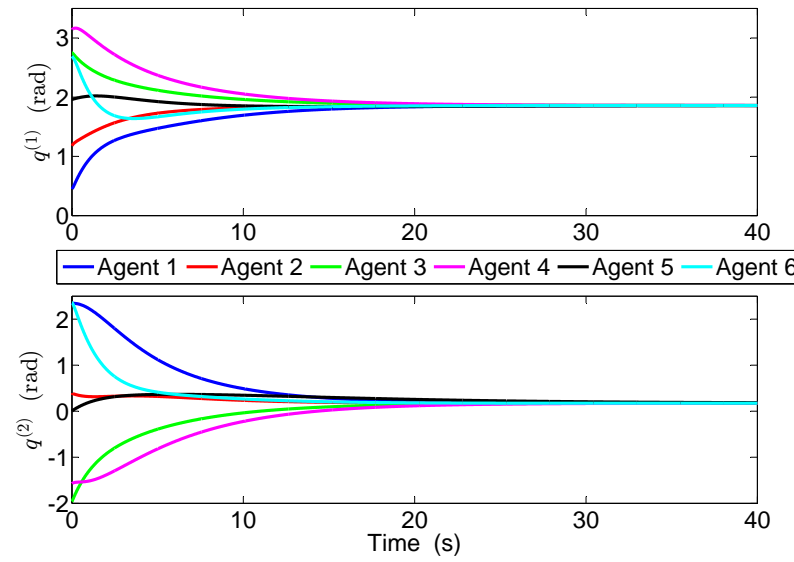

Figure 7. Plot of generalised coordinates vs. time, with $\mu \boldsymbol{K}_{i}=5 \boldsymbol{I}_{2}$
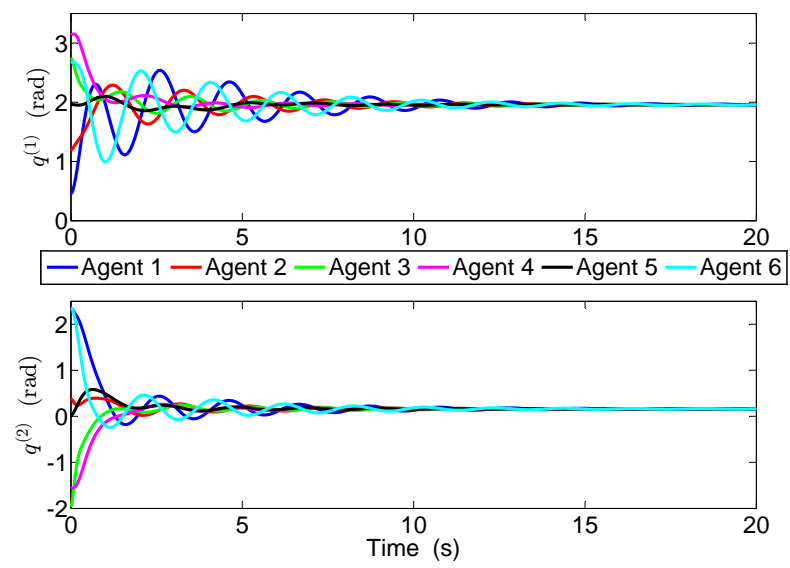

Figure 8. Plot of generalised coordinates vs. time, with each $a_{i j}$ multiplied by 2
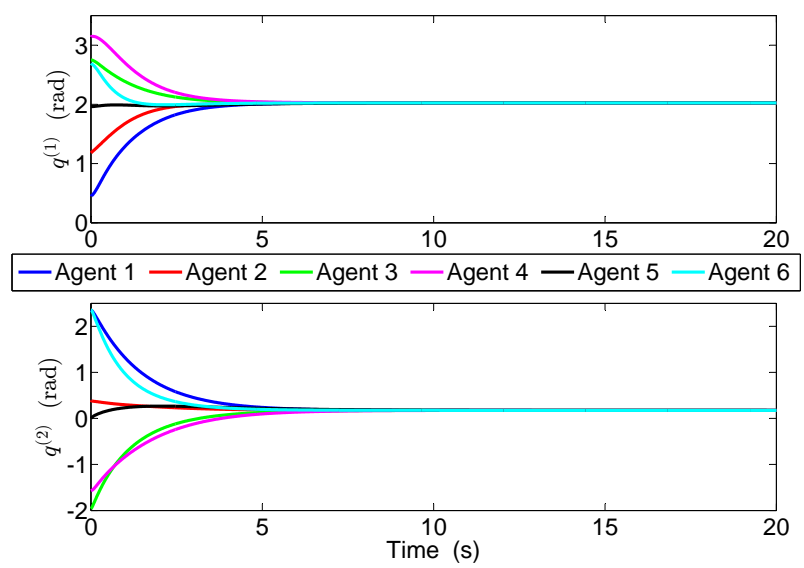

Figure 9. Plot of generalised coordinates vs. time, using the controller (52). 


\section{CONCLUSION}

A distributed model-independent algorithm is proposed in this paper which allows a directed network of agents with Euler-Lagrange dynamics to achieve leaderless consensus, and the network is required to be strongly connected. To ensure stability, a control gain scalar and a control gain matrix are designed to satisfy several lower bounding inequalities. These bounds require limited knowledge of global quantities associated with the networked system and so algorithm design is centralised while the algorithm is distributed in execution (knowledge of these quantities is reasonable). One of the required quantities is a bound on the possible initial conditions; this requirement means the algorithm is semi-globally stable. We use the bound on the initial conditions to derive a not necessarily tight upper bound on the trajectory of the networked system, and show that the bound holds for all time. We conclude that the leaderless consensus objective is achieved exponentially fast, at a computable minimum rate. Simulations are provided to show the algorithm's effectiveness and highlight remarks made about convergence speed and stability. Future work will consider time-delays in the network, switching topologies and relaxation of the graphical constraint from strongly connected to a directed spanning tree. A second direction of future work is to consider a gain-scheduling-like process to yield less conservative values of the control gain scalar.

\section{APPENDIX}

To show that there holds $\boldsymbol{G}>\boldsymbol{H}>\boldsymbol{L}>0$, it is sufficient to show that $\boldsymbol{G}>\boldsymbol{H}$ and $\boldsymbol{H}>\boldsymbol{L}$. The conclusion $\boldsymbol{L}>0$ is obtained from the arguments leading up to, and including the inequality (28). Observe that showing $\boldsymbol{H}>\boldsymbol{L}$ is equivalent to showing $\boldsymbol{H}-\boldsymbol{L}>0$. Notice that $\boldsymbol{\Gamma}_{p} \boldsymbol{M}-\left(\underline{\gamma} k_{\underline{m}}-\right.$ $\delta) \boldsymbol{I}_{n p}>0$. Taking this into consideration, Theorem 1 then implies that

$$
\boldsymbol{H}-\boldsymbol{L}=\left[\begin{array}{cc}
\frac{1}{2} \widetilde{\boldsymbol{T}}_{p}^{\top}\left(\widetilde{\mathcal{L}} \otimes \boldsymbol{I}_{p}\right) \widetilde{\boldsymbol{T}}_{p}-\frac{1}{4} \lambda_{2}(\widetilde{\mathcal{L}}) \boldsymbol{I}_{(n-1) p} & \frac{1}{2} \mu^{-1} \widetilde{\boldsymbol{T}}_{p}^{\top}\left[\boldsymbol{\Gamma}_{p} \boldsymbol{M}-\left(\underline{\gamma} k_{\underline{m}}-\delta\right) \boldsymbol{I}_{n p}\right] \\
\frac{1}{2} \mu^{-1}\left[\boldsymbol{M} \boldsymbol{\Gamma}_{p}-\left(\underline{\gamma} k_{\underline{m}}-\delta\right) \boldsymbol{I}_{n p}\right] \widetilde{\boldsymbol{T}}_{p} & \frac{1}{2} \boldsymbol{\Gamma}_{p} \boldsymbol{M}-\frac{1}{2}\left(\underline{\gamma} k_{\underline{m}}-\delta\right) \boldsymbol{I}_{n p}
\end{array}\right]>0
$$

is satisfied if there holds

$$
\frac{1}{2} \widetilde{\boldsymbol{T}}_{p}^{\top}\left(\widetilde{\mathcal{L}} \otimes \boldsymbol{I}_{p}\right) \widetilde{\boldsymbol{T}}_{p}-\frac{1}{4} \lambda_{2}(\widetilde{\mathcal{L}}) \boldsymbol{I}_{(n-1) p}-\frac{1}{2} \mu^{-1} \widetilde{\boldsymbol{T}}_{p}^{\top}\left[\boldsymbol{\Gamma}_{p} \boldsymbol{M}-\left(\underline{\gamma} k_{\underline{m}}-\delta\right) \boldsymbol{I}_{n p}\right] \widetilde{\boldsymbol{T}}_{p}>0
$$

Using Lemma 4 we obtain the following inequality

$$
\begin{aligned}
\lambda_{\max }\left(\widetilde{\boldsymbol{T}}_{p}^{\top}\left[\boldsymbol{\Gamma}_{p} \boldsymbol{M}-\left(\underline{\gamma} k_{\underline{m}}-\delta\right) \boldsymbol{I}_{n p}\right] \widetilde{\boldsymbol{T}}_{p}\right) & \leq \lambda_{\max }\left[\boldsymbol{\Gamma}_{p} \boldsymbol{M}-\left(\underline{\gamma} k_{\underline{m}}-\delta\right) \boldsymbol{I}_{n p}\right] \\
& \leq \bar{\gamma} k_{\bar{M}}-\left(\underline{\gamma} k_{\underline{m}}-\delta\right)<\bar{\gamma} k_{\bar{M}}
\end{aligned}
$$

Using Corollary 1, expressions (1) and (4), it is then straightforward to compute that (60) is implied by $\frac{1}{4} \lambda_{2}(\widetilde{\mathcal{L}})-\frac{1}{2} \mu^{-1} \bar{\gamma} k_{\bar{M}}>0$ and holds for all $\mu \geq \mu_{5}^{*}$ where $\mu_{5}^{*}$ is defined in (36). We have therefore established that $\boldsymbol{H}>\boldsymbol{L}$.

Next, consider $\boldsymbol{G}-\boldsymbol{H}$ as shown in (62). Because $\left(\bar{\gamma} k_{\bar{M}}+\delta\right) \boldsymbol{I}_{n p}-\boldsymbol{\Gamma}_{p} \boldsymbol{M}>0$, Theorem 1 indicates that

$$
\boldsymbol{G}-\boldsymbol{H}=\left[\begin{array}{cc}
\lambda_{\max }(\widetilde{\mathcal{L}}) \boldsymbol{I}_{(n-1) p}-\frac{1}{2} \widetilde{\boldsymbol{T}}_{p}^{\top}\left(\widetilde{\mathcal{L}} \otimes \boldsymbol{I}_{p}\right) \widetilde{\boldsymbol{T}}_{p} & \frac{1}{2} \mu^{-1} \widetilde{\boldsymbol{T}}_{p}^{\top}\left[\left(\bar{\gamma} k_{\bar{M}}+\delta\right) \boldsymbol{I}_{n p}-\boldsymbol{\Gamma}_{p} \boldsymbol{M}\right] \\
\frac{1}{2} \mu^{-1}\left[\left(\bar{\gamma} k_{\bar{M}}+\delta\right) \boldsymbol{I}_{n p}-\boldsymbol{M} \boldsymbol{\Gamma}_{p}\right] \widetilde{\boldsymbol{T}}_{p} & \frac{1}{2}\left(\bar{\gamma} k_{\bar{M}}+\delta\right) \boldsymbol{I}_{n p}-\frac{1}{2} \boldsymbol{\Gamma}_{p} \boldsymbol{M}
\end{array}\right]>0
$$

is implied by

$$
\lambda_{\max }(\widetilde{\mathcal{L}}) \boldsymbol{I}_{(n-1) p}-\frac{1}{2} \widetilde{\boldsymbol{T}}_{p}^{\top}\left(\widetilde{\mathcal{L}} \otimes \boldsymbol{I}_{p}\right) \widetilde{\boldsymbol{T}}_{p}-\frac{1}{2} \mu^{-1} \widetilde{\boldsymbol{T}}_{p}^{\top}\left[\left(\bar{\gamma} k_{\bar{M}}+\delta\right) \boldsymbol{I}_{n p}-\boldsymbol{M} \boldsymbol{\Gamma}_{p}\right] \widetilde{\boldsymbol{T}}_{p}>0
$$

Similar to (61), the following inequality holds

$$
\begin{aligned}
\lambda_{\max }\left(\widetilde{\boldsymbol{T}}_{p}^{\top}\left[\left(\bar{\gamma} k_{\bar{M}}+\delta\right) \boldsymbol{I}_{n p}-\boldsymbol{M} \boldsymbol{\Gamma}_{p}\right] \widetilde{\boldsymbol{T}}_{p}\right) & \leq \lambda_{\max }\left[\left(\bar{\gamma} k_{\bar{M}}+\delta\right) \boldsymbol{I}_{n p}-\boldsymbol{M} \boldsymbol{\Gamma}_{p}\right] \\
& \leq\left(\bar{\gamma} k_{\bar{M}}+\delta\right)-\underline{\gamma} k_{\underline{m}}<\bar{\gamma} k_{\bar{M}}
\end{aligned}
$$


Straightforward computation will then shown that (63) is implied by $\frac{1}{2} \lambda_{\max }(\widetilde{\mathcal{L}})-\frac{1}{2} \mu^{-1} \bar{\gamma} k_{\bar{M}}>0$, and is satisfied for all $\mu \geq \mu_{5}^{*}$, and thus $\boldsymbol{G}>\boldsymbol{H}$.

\section{ACKNOWLEDGEMENT}

This work was supported by the Australian Research Council (ARC) under the ARC grants DP-130103610 and DP-160104500, by the National Natural Science Foundation of China (grant 61375072), and by Data61CSIRO (formerly NICTA).

\section{REFERENCES}

1. Cao Y, Yu W, Ren W, Chen G. An Overview of Recent Progress in the Study of Distributed Multi-Agent Coordination. IEEE Transactions on Industrial Informatics Feb 2013; 9(1):427-438, doi:10.1109/TII.2012. 2219061.

2. Knorn S, Chen Z, Middleton R. Overview: Collective control of multi-agent systems. IEEE Transactions on Control of Network Systems 2015; PP(99):1-1, doi:10.1109/TCNS.2015.2468991.

3. Ren W, Cao Y. Distributed Coordination of Multi-agent Networks: Emergent Problems, Models and Issues. Springer London, 2011.

4. Ren W, Beard R. Distributed Consensus in Multi-vehicle Cooperative Control: Theory and Applications. Springer London, 2007.

5. Chung SJ, Slotine JJ. Cooperative Robot Control and Concurrent Synchronization of Lagrangian Systems. IEEE Transactions on Robotics 2009; 25(3):686-700.

6. Meng Z, Lin Z. Distributed Finite-time Cooperative Tracking of Networked Lagrange Systems via Local Interactions. American Control Conference (ACC), Montréal, Canada, IEEE, 2012; 4951-4956.

7. Chen G. Cooperative controller design for synchronization of networked uncertain Euler-Lagrange systems. International Journal of Robust and Nonlinear Control 2015; 25(11):1721-1738, doi:10.1002/rnc.3172.

8. Spong MW, Hutchinson S, Vidyasagar M. Robot Modeling and Control, vol. 3. Wiley New York, 2006.

9. Kelly R, Davila VS, Perez JAL. Control of Robot Manipulators in Joint Space. Springer Science \& Business Media London, 2006

10. Meng Z, Lin Z, Ren W. Leader-Follower Swarm Tracking for Networked Lagrange Systems. Systems \& Control Letters 2012; 61(1):117-126.

11. Mei J, Ren W, Ma G. Distributed Containment Control for Lagrangian Networks With Parametric Uncertainties Under a Directed Graph. Automatica 2012; 48(4):653-659.

12. Mei J, Ren W, Chen J, Ma G. Distributed adaptive coordination for multiple lagrangian systems under a directed graph without using neighbors' velocity information. Automatica 2013; 49(6):1723-1731.

13. Chen G, Lewis FL. Distributed Adaptive Tracking Control for Synchronization of Unknown Networked Lagrangian Systems. IEEE Transactions on Systems, Man, and Cybernetics, Part B: Cybernetics 2011; 41(3):805-816.

14. Nuno E, Ortega R, Basanez L, Hill D. Synchronization of Networks of Nonidentical Euler-Lagrange Systems with Uncertain Parameters and Communication Delays. IEEE Transactions on Automatic Control 2011; 56(4):935-941.

15. Meng Z, Dimarogonas DV, Johansson KH. Leader-Follower Coordinated Tracking of Multiple Heterogeneous Lagrange Systems Using Continuous Control. IEEE Transactions on Robotics 2014; 30(3):739-745.

16. Ghapani S, Mei J, Ren W, Song Y. Fully distributed flocking with a moving leader for Lagrange networks with parametric uncertainties. Automatica 2016; 67:67-76.

17. Abdessameud A, Tayebi A, Polushin IG. On the Leader-Follower Synchronization of Euler-Lagrange Systems. Proceedings of 54th IEEE Conference on Decision and Control, IEEE, 2015; 1054-1059.

18. Atinc G, Stipanovic DM. Cooperative Collision-Free Control of Lagrangian Multi-Agent Formations. American Control Conference (ACC), San Francisco, USA, IEEE, 2011; 2801-2806.

19. Ren W. Distributed Leaderless Consensus Algorithms for Networked Euler-Lagrange Systems. International Journal of Control 2009; 82(11):2137-2149.

20. Meng Z, Yang T, Shi G, Dimarogonas DV, Hong Y, Johansson KH. Set Target Aggregation of Multiple Mechanical Systems. 2014 IEEE 53rd Annual Conference on Decision and Control, IEEE, 2014; 6830-6835.

21. Nuno E, Sarras I, Basanez L. Consensus in Networks of Nonidentical Euler-Lagrange Systems using P+ d Controllers. IEEE Transactions on Robotics 2013; 29(6):1503-1508.

22. Tavasoli A, Taghvaee S, Eghtesad M. Flocking of a Team of Lagrangian Agents. IEEE International Conference on Robotics and Biomimetics (ROBIO), Guilin, China, IEEE, 2009; 1433-1438.

23. Mei J, Ren W, Ma G. Distributed Coordinated Tracking With a Dynamic Leader for Multiple Euler-Lagrange Systems. IEEE Transactions on Automatic Control 2011; 56(6):1415-1421.

24. Zhao Y, Duan Z, Wen G. Distributed finite-time tracking of multiple Euler-Lagrange systems without velocity measurements. International Journal of Robust and Nonlinear Control 2015; 25(11):1688-1703, doi:10.1002/rnc. 3170 .

25. Klotz JR, Kan Z, Shea JM, Pasiliao EL, Dixon WE. Asymptotic Synchronization of a Leader-Follower Network of Uncertain Euler-Lagrange Systems. IEEE Transactions on Control of Network Systems June 2015; 2(2):174-182, doi:10.1109/TCNS.2014.2378875.

26. Feng Z, Hu G, Ren W, Dixon WE, Mei J. Distributed Coordination of Multiple Unknown Euler-Lagrange Systems. IEEE Transactions on Control of Network Systems 2016; PP(99):1-1, doi:10.1109/TCNS.2016.2578039.

27. Klotz JR, Cheng TH, Dixon WE. Robust containment control in a leader-follower network of uncertain EulerLagrange systems. International Journal of Robust and Nonlinear Control 2016; doi:10.1002/rnc.3535. 
28. Hokayem PF, Stipanović DM, Spong MW. Coordination and Collision Avoidance for Lagrangian Systems With Disturbances. Applied Mathematics and Computation 2010; 217(3):1085-1094.

29. Chopra N. Output Synchronization on Strongly Connected Graphs. IEEE Transactions on Automatic Control Nov 2012; 57(11):2896-2901, doi:10.1109/TAC.2012.2193704.

30. Ye M, Yu C, Anderson BDO. Model-Independent Rendezvous of Euler-Lagrange Agents on Directed Networks. Proceedings of IEEE 54th Annual Conference on Decision and Control, Osaka, Japan, 2015; 3499-3505.

31. Horn RA, Johnson CR. Matrix Analysis. Cambridge University Press, New York, 2012.

32. Zhang H, Lewis FL, Qu Z. Lyapunov, Adaptive, and Optimal Design Techniques for Cooperative Systems on Directed Communication Graphs. IEEE Transactions on Industrial Electronics July 2012; 59(7):3026-3041, doi: 10.1109/TIE.2011.2160140.

33. Olfati-Saber R, Murray R. Consensus Problems in Networks of Agents With Switching Topology and Time-Delays. IEEE Transactions on Automatic Control Sept 2004; 49(9):1520-1533, doi:10.1109/TAC.2004.834113.

34. Harville DA. Matrix Algebra From a Statistician's Perspective. Springer-Verlag New York, 2008.

35. Dimarogonas DV, Frazzoli E, Johansson KH. Distributed Event-Triggered Control for Multi-Agent Systems. IEEE Transactions on Automatic Control 2012; 57(5):1291-1297.

36. Liu Q, Ye M, Qin J, Yu C. Event-based leader-follower consensus for multiple Euler-Lagrange systems with parametric uncertainties. to appear in IEEE 55th Annual Conference on Decision and Control.

37. Huang N, Duan Z, Zhao Y. Distributed consensus for multiple Euler-Lagrange systems: An event-triggered approach. Science China: Technological Sciences January 2016; 59:33-44.

38. Song Q, Liu F, Cao J, Yu W. M-Matrix Strategies for Pinning-Controlled Leader-Following Consensus in Multiagent Systems With Nonlinear Dynamics. IEEE Transactions on Cybernetics Dec 2013; 43(6):1688-1697, doi:10.1109/TSMCB.2012.2227723.

39. Wen G, Duan Z, Chen G, Yu W. Consensus Tracking of Multi-Agent Systems With Lipschitz-Type Node Dynamics and Switching Topologies. IEEE Transactions on Circuits and Systems I: Regular Papers 2014; 61(2):499-511.

40. Fan MC, Chen Z, Zhang HT. Semi-Global Consensus of Nonlinear Second-Order Multi-Agent Systems With Measurement Output Feedback. IEEE Transactions on Automatic Control 2014; 59(8):2222-2227. 